\title{
Leprosy drug clofazimine activates peroxisome proliferator-activated receptor- $\gamma$ and synergizes with imatinib to inhibit chronic myeloid leukemia cells
}

\begin{abstract}
Harish Kumar, ${ }^{1}$ Sourav Chattopadhyay, ${ }^{1,2}$ Nabanita Das, ${ }^{1}$ Sonal Shree, ${ }^{3}$ Dinesh Patel, ${ }^{4}$ Jogeswar Mohapatra, ${ }^{4}$ Anagha Gurjar, ${ }^{1,2}$ Sapana Kushwaha, ${ }^{1}$ Abhishek Kumar Singh, ${ }^{1}$ Shikha Dubey, ${ }^{3}$ Kiran Lata, ${ }^{3}$ Rajesh Kushwaha, ${ }^{5}$ Riyazuddin Mohammed, ${ }^{6}$ Krishnarup Ghosh Dastidar, ${ }^{4}$ Namrata Yadav, ${ }^{4}$ Achchhe Lal Vishwakarma, ${ }^{7}$ Jiaur Rahaman Gayen, ${ }^{6,2}$ Sanghamitra Bandyopadhyay, ${ }^{5}$ Abhijit Chatterjee, ${ }^{4}$ Mukul Rameshchandra Jain, ${ }^{4}$ Anil Kumar Tripathi, ${ }^{8}$ Arun Kumar Trivedi, ${ }^{1,2}$ Naibedya Chattopadhyay, ${ }^{9,2}$ Ravishankar Ramachandran $^{2,3}$ and Sabyasachi Sanyal ${ }^{1,2}$

${ }^{1}$ Division of Biochemistry, CSIR-Central Drug Research Institute, Lucknow; ${ }^{2}$ AcSIR, CSIRCentral Drug Research Institute Campus, Lucknow; ${ }^{3}$ Division of Molecular and Structural Biology, CSIR-Central Drug Research Institute, Lucknow; ${ }^{4}$ Zydus Research Center, Moraiya, Ahmedabad, Gujarat; ${ }^{5}$ Developmental Toxicology Laboratory, Systems Toxicology and Health Risk Assessment Group, CSIR-Indian Institute of Toxicology Research, Lucknow; ${ }^{6}$ Pharmacokinetics and Metabolism Division, CSIR-Central Drug Research Institute, Lucknow; ' Sophisticated Analytical Instrument Facility, CSIR-Central Drug Research Institute, Lucknow; ' Department of Clinical Hematology and Medical Oncology, King George's Medical University, Lucknow, Uttar Pradesh and 'Division of Endocrinology, CSIRCentral Drug Research Institute, Lucknow, India
\end{abstract}

L eukemia stem cells contribute to drug-resistance and relapse in chronic myeloid leukemia (CML) and BCR-ABL1 inhibitor monotherapy fails to eliminate these cells, thereby necessitating alternate therapeutic strategies for patients CML. The peroxisome proliferator-activated receptor$\gamma(\mathrm{PPAR} \gamma)$ agonist pioglitazone downregulates signal transducer and activator of transcription 5 (STAT5) and in combination with imatinib induces complete molecular response in imatinib-refractory patients by eroding leukemia stem cells. Thiazolidinediones such as pioglitazone are, however, associated with severe side effects. To identify alternate therapeutic strategies for CML we screened Food and Drug Administration-approved drugs in K562 cells and identified the leprosy drug clofazimine as an inhibitor of viability of these cells. Here we show that clofazimine induced apoptosis of blood mononuclear cells derived from patients with CML, with a particularly robust effect in imatinib-resistant cells. Clofazimine also induced apoptosis of $\mathrm{CD} 34^{+} 38$ progenitors and quiescent $\mathrm{CD} 34^{+}$cells from $\mathrm{CML}$ patients but not of hematopoietic progenitor cells from healthy donors. Mechanistic evaluation revealed that clofazimine, via physical interaction with PPAR $\gamma$, induced nuclear factor $\kappa \mathrm{B}$-p65 proteasomal degradation, which led to sequential myeloblastoma oncoprotein and peroxiredoxin 1 downregulation and concomitant induction of reactive oxygen species-mediated apoptosis. Clofazimine also suppressed STAT5 expression and consequently downregulated stem cell maintenance factors hypoxia-inducible factor- $1 \alpha$ and $-2 \alpha$ and Cbp/P300 interacting transactivator with Glu/Asp-rich carboxy-terminal domain 2 (CITED2). Combining imatinib with clofazimine caused a far superior synergy than that with pioglitazone, with clofazimine reducing the half maximal inhibitory concentration $\left(\mathrm{IC}_{50}\right)$ of imatinib by $>4$ logs and remarkably eroding quiescent $\mathrm{CD} 34^{+}$cells. In a K562 xenograft study clofazimine and imatinib co-treatment showed more robust efficacy than the individual treatments. We propose clinical evaluation of clofazimine in imatinibrefractory CML.

\section{Ferrata Storti Foundation}

Haematologica 2020

Volume 105(4):971-986

\section{Correspondence:}

SABYASACHI SANYAL

sanyal@cdri.res.in

Received: April 11, 2019

Accepted: July 12, 2019.

Pre-published: August 1, 2019.

doi:10.3324/haematol.2018.194910

Check the online version for the most updated information on this article, online supplements, and information on authorship \& disclosures: www.haematologica.org/content/105/4/971

\section{(C)2020 Ferrata Storti Foundation}

Material published in Haematologica is covered by copyright. All rights are reserved to the Ferrata Storti Foundation. Use of published material is allowed under the following terms and conditions:

https://creativecommons.org/licenses/by-nc/4.0/legalcode. Copies of published material are allowed for personal or internal use. Sharing published material for non-commercial purposes is subject to the following conditions:

https://creativecommons. org//icenses/by-nc/4.0/legalcode sect. 3. Reproducing and sharing published material for commercial purposes is not allowed without permission in writing from the publisher. 


\section{Introduction}

The therapy of chronic myeloid leukemia (CML) has seen tremendous advances following the discovery of imatinib and other BCR-ABL1 tyrosine kinase inhibitors. However, complete molecular response, defined as undetectable $B C R-A B L 1$ transcripts, is not achieved in the majority of patients. ${ }^{1}$ Resistance to tyrosine kinase inhibitors may occur due to $B C R$ - $A B L 1$ mutations; however, in approximately $50 \%$ of the cases BCR-ABL1-independent mechanisms, including tyrosine kinase inhibitorrefractory leukemia stem cells (LSC), contribute to resistance and recurrence. ${ }^{1}$ Therefore therapeutic approaches capable of overcoming resistance to tyrosine kinase inhibitors are needed. Peroxisome proliferator-activated receptor- $\gamma$ (PPAR $\gamma$ ) agonists, pioglitazone in particular, were reported to erode quiescent LSC by targeting signal transducer and activator of transcription 5 (STAT5) expression. ${ }^{1,2}$ Unfortunately, pioglitazone increases the risk of bladder cancer. ${ }^{3}$ Although rosiglitazone has not been found to increase the incidence of bladder cancer, it is associated with severe cardiovascular risks. ${ }^{4}$

To identify new therapeutic strategies we screened 800 Food amd Drug Administration-approved drugs for their anti-CML efficacy in the K562 cell line and identified clofazimine as a potent inhibitor of viability. Clofazimine, a riminophenazine leprosy drug, is also effective against multidrug-resistant tuberculosis ${ }^{5}$ and imparts its anti-bacterial actions by generating reactive oxygen species (ROS), particularly superoxides and hydrogen peroxide $\left(\mathrm{H}_{2} \mathrm{O}_{2}\right){ }^{6}$ Clofazimine also displays anti-inflammatory properties that are important for its suppression of leprosy-associated immune reactions. ${ }^{6}$ Additionally, clofazimine was shown to be effective against various autoimmune diseases, including discoid lupus erythematosus, Crohn disease, ulcerative colitis, psoriasis, Meischer granuloma and graft-versus-host disease.? Clofazimine is reported to exert its immunomodulatory activities by blocking KV1.3 voltage-gated potassium channels ${ }^{7}$ and thereby inhibiting chronic lymphocytic leukemia cells.,9

Here we report the anti-CML efficacy of clofazimine in cells lacking KV1.3, ${ }^{8,10}$ and show that clofazimine exerted its effects by binding to PPAR $\gamma$. Clofazimine not only suppressed STAT5 expression by modulating PPAR $\gamma$ transcriptional activity but also regulated a novel signaling cascade by increasing PPAR $\gamma$-mediated p65 nuclear factor kappa B (NFkB) degradation, which caused transcriptional downregulation of cellular myeloblastoma oncoprotein (MYB), leading to suppression of peroxiredoxin 1 (PRDX1) expression and consequent induction of ROS-mediated apoptosis and differentiation.

\section{Methods}

\section{Cell lines and human primary cells}

K562 (CCL-243), HL-60 (CCL-240), U937 (CRL-1593.2), and HEK-293 (CRL-1573) cells were from the American Type Culture Collection (ATCC; Manassas, VA, USA) and were maintained as per ATCC instructions. Peripheral blood samples were obtained from BCR-ABL1+ CML patients (newly diagnosed, imatinib-resistant and imatinib responders), and healthy donors from King George's Medical University (Clinical Hematology and Medical Oncology Division, Lucknow, India) following ethical approval (approval n. 1638/R. Cell-12) as per institutional ethical guidelines after written consent (patients' details in Online Supplementary Table S1). Peripheral blood mononuclear cells were isolated on a Percoll (Sigma) density gradient by centrifugation. All analyses of peripheral blood mononuclear cells were conducted on gated mononuclear cells excluding lymphocytes.

\section{Other methods}

Chemicals, antibodies, plasmid information and experimental procedures are detailed in the Online Supplementary Methods.

\section{Statistical analysis}

Data are expressed as the mean \pm standard error of mean of three independent experiments, unless otherwise indicated. Statistical analyses were performed using GraphPad Prism 5.0. An unpaired two-tailed Student $t$-test or Mann-Whitney U test was used to compare two groups. Equality of variances was assessed by the F-test. Statistical analyses involving more than two groups were performed by one- or two-way analysis of variance followed by the Bonferroni post-test, or Kruskal-Wallis test followed by the Dunn test. For intra-group variances, we used the Levene median test (equal sample size; using XLSTAT) or Bartlett test (unequal sample size). $P<0.05$ was accepted as statistically significant.

\section{Results}

\section{Clofazimine induces apoptosis and differentiation in chronic myeloid leukemia cells}

In a screening in K562 cells, we identified clofazimine as a potent inhibitor of viability. Clofazimine has been reported to induce cytotoxicity by targeting KV1.3.-9 Intriguingly, although K562 does not express KV1.3,10 (Online Supplementary Figure S1A), clofazimine reduced the viability of these cells with a pharmacologically relevant half maximal inhibitory concentration (IC $\mathrm{I}_{50}$ ) of $5.85 \mu \mathrm{M}$ (Figure 1A). The human plasma $\mathrm{C}_{\max }$ of clofazimine is 0.4$4 \mathrm{mg} / \mathrm{L}$, equivalent to $0.84-8.4 \mu \mathrm{M} .^{6,11-13}$ The loss of viability was due to apoptosis, as demonstrated by annexin $\mathrm{V}$ staining (Figure 1B, Online Supplementary Figure S1B), poly (ADP-ribose) polymerase (PARP) cleavage (Figure 1C) and terminal deoxynucleotidyl transferase dUTP nick end labeling (TUNEL) (Online Supplementary Figure S1C). Clofazimine induced cytochrome $\mathrm{C}$ release and activated caspase -3 and -9 but not -8 (Figure 1D), suggesting mitochondria-mediated apoptosis, which was consistent with decreased B-cell lymphoma 2 (BCL-2) and increased BAX expression (Figure 1D). Clofazimine also induced apoptosis in peripheral blood mononuclear cells from patients with chronic phase CML (CP-CML cells; one newly diagnosed patient was in accelerated phase, one imatinibresponder was in blast crisis) with an efficacy similar to that of cells from patients with newly diagnosed CML and imatinib-responders but higher than that of imatinib and dasatinib in imatinib-resistant cells, while it did not affect healthy donor cells (Figure 1E, Online Supplementary Figure $S 1 D$ ). Among the 21 imatinib-resistant patients (Figure $1 \mathrm{E})$, seven harbored the following $B C R-A B L 1$ mutations; M244V ( $n=1), Y 253 H(n=2), M 351 \mathrm{~T}(\mathrm{n}=3)$ and F359V $(n=1)$; clofazimine showed efficacy in all cases (Figure 1F; upper panel). A separate analysis of apoptosis in imatinibresistant patients without $B C R$-ABL1 mutations (from Figure 1E) also showed significant clofazimine-induced apoptosis ( $\mathrm{n}=6$ : vehicle, imatinib, clofazimine; $\mathrm{n}=5$; dasatinib. Figure 1F; lower panel), indicating that clofazimine- 
induced apoptosis in imatinib-resistant cells is independent of $B C R-A B L 1$ mutations.

We next assessed whether clofazimine inhibited LSC. Increased aldehyde dehydrogenase activity is a hallmark of cancer stem cells ${ }^{14}$ and clofazimine reduced it in imatinib-resistant CP-CML cells at par with the positive control salinomycin (Figure 1G, Online Supplementary Figure $S 2 A)$. Clofazimine also reduced colony formation in CML $\mathrm{CD} 34^{+}$cells (Figure $1 \mathrm{H}$, Online Supplementary Figure S2C). To further confirm its anti-LSC efficacy we treated purified CML CD34+ cells with clofazimine and analyzed them by CD34 or annexin V staining. Clofazimine reduced the $\mathrm{CD} 34^{+}$population and induced apoptosis in these cells (Figure 1I, Online Supplementary Figure S2B). Treating CML CD34+ cells with clofazimine and analyzing them by CD34, CD38 and annexin V co-staining revealed that clofazimine induced apoptosis in both committed $\mathrm{CD}_{3} 4^{+} 38^{+}$and primitive $\mathrm{CD} 34^{+} 38^{-}$progenitor cells (Figure 1J). Clofazimine however, caused $<5 \%$ loss in viability and no apoptosis in hematopoietic progenitor cells from healthy donors (Figure 1K, L, Online Supplementary Figure S2D) indicating that it specifically targets LSC. Clofazimine's effects in CML CD34+ cells were not routed through KV1.3 as KV1.3 transcript was undetectable in CD34+-enriched CP-CML cells ( $\mathrm{n}=7$; Online Supplementary Figure S3).

We next investigated whether clofazimine induced differentiation at sub-lethal concentrations. In K562 cells that predominantly undergo erythroid or megakaryocytic differentiation upon various stimuli, clofazimine induced a megakaryocyte-like phenotype characterized by increased cellular size, nuclear to cytoplasmic ratio, vacuolation, and lobulated nuclei (Online Supplementary Figure S4A). Consistently, clofazimine increased megakaryocytic surface markers CD61 and CD41 in K562 (Online Supplementary Figure S4B-D) and CML (one newly diagnosed patient was in accelerated phase, one imatinibresistant patient was in blast crisis) cells (Figure $1 \mathrm{M}$, Online Supplementary Figure S4E-F). Clofazimine treatment in $\mathrm{CML} \mathrm{CD} 4^{+}$cells followed by May-Grünwald-Giemsa staining revealed increased monocyte-like morphology (Figure 1N, Online Supplementary Figure S4G). Concurrently, expression of the monocyte/macrophage differentiation marker CD11b was increased in clofazimine-treated $\mathrm{CD}^{+}{ }^{+}$cells (Figure 10, Online Supplementary Figure S4H). Furthermore, clofazimine also induced CD61 in $\mathrm{CD}^{+} 4^{+}$cells (Figure 1P, Online Supplementary Figure S4H).

\section{Apoptosis- and differentiation-inducing effects of clofazimine are associated with enhanced reactive oxygen species and decreased PRDX1 expression}

We next asked how clofazimine exerts its action and decided to address its differentiation-inducing activity first. Megakaryocytic differentiation in K562 cells is typically associated with prolonged extracellular signal-regu-
A

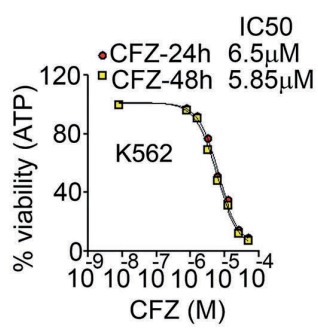

$E$

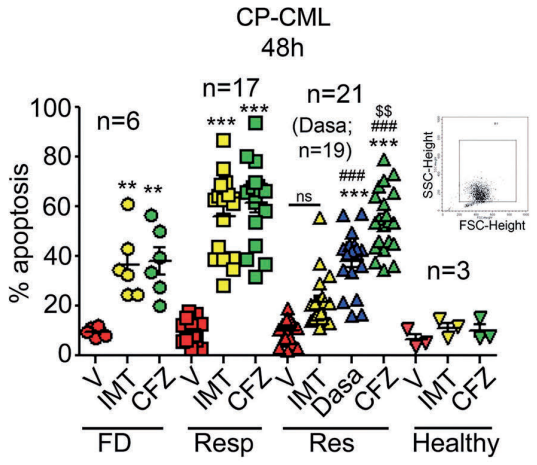

B

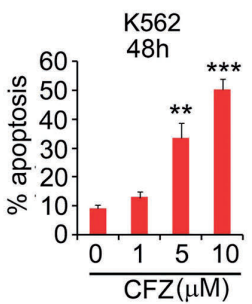

C

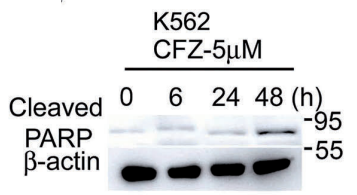

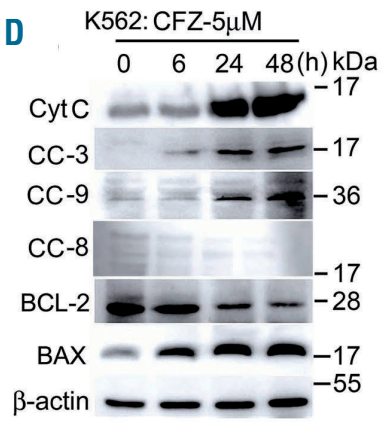

H

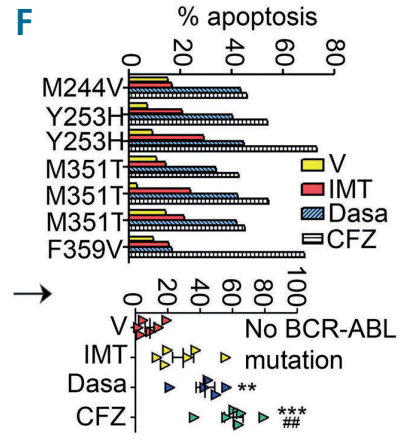

G

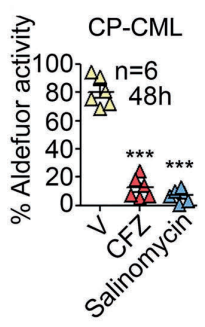

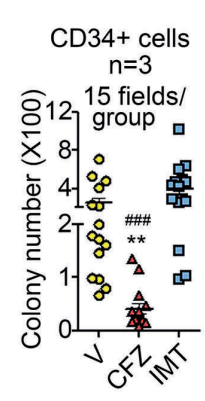

Figure 1. Clofazimine induces apoptosis and differentiation in K562 and chronic phase chronic myeloid leukemia cells and reduces leukemia stem cell load. (A, B) Clofazimine (CFZ) reduces K562 cell viability and induces apoptosis. (A) CFZ dose response, as determined by a CellTiter-Glo assay. (B) Apoptosis ( $\mathrm{n}=3$; representative dot plot in Online Supplementary Figure S1B). (C) Poly (ADP-ribose) polymerase cleavage in K562 cells. (D) CFZ induces cytochrome C release, caspase cleavage, BAX expression and suppresses BCL-2 in K562 cells. (E, F) CFZ (all drugs $5 \mu \mathrm{M}$ ) induces apoptosis in chronic phase chronic myeloid leukemia (CP-CML) cells (annexin/propidium iodide (PI) staining; dot plots in Online Supplementary Figure S1D). (F) Percentage apoptosis in cells from imatinib-resistant patients in Figure $1 \mathrm{E}$, harboring the indicated BCR-ABL1 mutations (upper panel) and CP-CML cells in which no BCR-ABL1 mutations were detected (lower panel). (G) CFZ (or salinomycin; both at a concentration of $5 \mu \mathrm{M}$ ) reduced aldehyde dehydrogenase activity, determined by percentage aldefluor activity in CP-CML cells (dot plots in Online Supplementary Figure S2A). (H) CFZ reduces the number of colony-forming cells in soft agar (images in Online Supplementary Figure S2C; imatinib $1 \mu$ M, CFZ 2.5 $\mu \mathrm{M})$. (continued on next page) 
lated kinase (ERK) activation. ${ }^{15,16}$ Interestingly, mitogenactivated protein kinase (MAPK) kinase inhibitor U0126 failed to inhibit clofazimine but not phorbol myristate acetate (PMA)-induced CD41 expression (Figure 2A, Online Supplementary Figure S5A). Consistently, PMA but not clofazimine induced ERK phosphorylation in K562 cells (Figure 2B). Apart from ERK, ROS induces megakaryocytic differentiation ${ }^{17-19}$ and determination of cellular ROS revealed that clofazimine significantly enhanced ROS production from $12 \mathrm{~h}$ onwards (Figure 2C, Online Supplementary Figure S5B). Clofazimine also enhanced mitochondrial superoxide (Figure $2 \mathrm{D}, \mathrm{E}$ ) and $\mathrm{H}_{2} \mathrm{O}_{2}$ (Figure $2 \mathrm{~F})$ and caused mitochondrial membrane depolarization (Figure 2G, Online Supplementary Figure S5C). Co-treatment with ROS scavengers and inhibitors revealed that $\alpha$ - tocopherol, which interacts with superoxides ${ }^{20}$ and also blocks $\mathrm{H}_{2} \mathrm{O}_{2}$ and peroxynitrite-mediated toxicity, ${ }^{21-23} \mathrm{com}-$ pletely abrogated clofazimine-induced cell death while the ROS scavenger $\mathrm{N}$-acetyl-L-cysteine and $\mathrm{H}_{2} \mathrm{O}_{2}$ decomposer catalase had lesser but significant effects, and the mitochondrial complex inhibitors rotenone and antimycin and the NADPH oxidase inhibitor diphenyleneiodonium were ineffective (Figure $2 \mathrm{H}$ ). $\alpha$-tocopherol also inhibited clofazimine-induced CD41 and CD61 expression (Figure 2I, Online Supplementary Figure S5D-F). These results indicate that clofazimine induced ROS-dependent cell death and differentiation.

Cancer cells, including cancer stem cells, display high levels of ROS coupled with increased antioxidants that help them detoxify ROS. ${ }^{24-27}$ We thus investigated whether
I

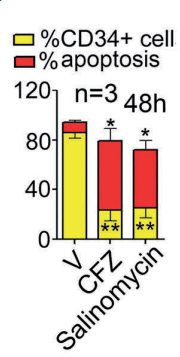

L

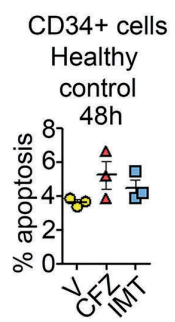

0

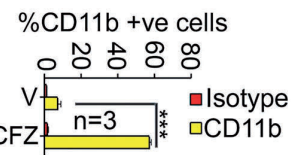

J

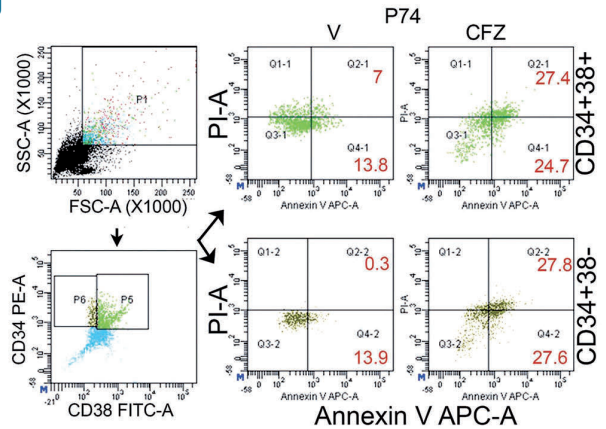

$96 \mathrm{~h}$

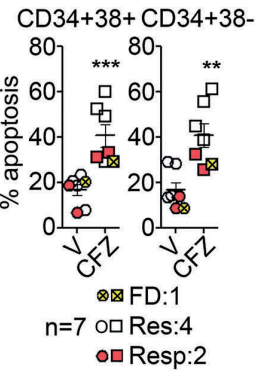

K

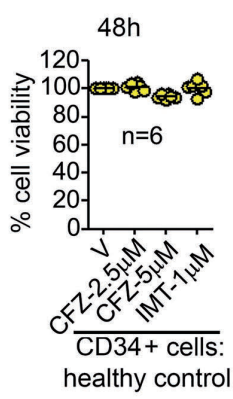

$n=3$
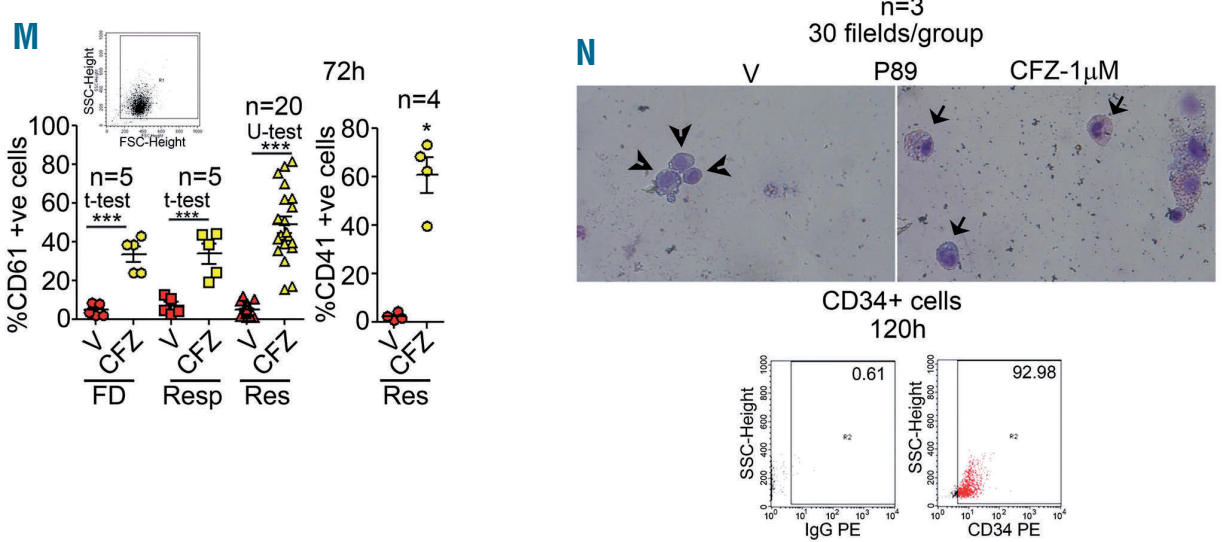

P $\quad \%$ CD61+ve cells

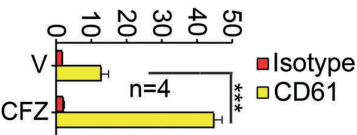

CD34+ cells: $72 \mathrm{~h}$

Figure 1. (continued from previous page) (I, J) CFZ induces apoptosis in CP-CML CD34+ cells. (I) CD34+ cells from imatinib-resistant patients were isolated using a CD34 microbead kit (Miltenyi Biotech) and were treated with 5 uM CFZ or salinomycin for $48 \mathrm{~h}$. Cells were then divided into two groups. One group was assessed by immunostaining for CD34 and the other group was stained with annexin V/PI and the cells were then analyzed by flow cytometry (dot plots in Online Supplementary Figure S2B). $(J)$ The CD34 $4^{+}$population from imatinib-resistant CP-CML cells was treated with CFZ $(2.5 \mu \mathrm{M})$ for $96 \mathrm{~h}$, stained with anti-CD34 and anti-CD38 antibodies and assessed for apoptosis. (K, L) CFZ does not affect viability of hematopoietic progenitors from healthy controls. (K) Cell viability, determined by a CellTiter-Glo assay. (L) Apoptosis by annexin V staining (dot plots in Online Supplementary Figure S2D). (M) CFZ (1 uM) induces CD61 and CD41 in CP-CML cells (dot plots in Online Supplementary Figure $\mathrm{S} 4 \mathrm{E}, \mathrm{F}$ ). (N) CFZ induces monocyte-like morphology in CD34+ cells (cropped images shown; corresponding original images in Online Supplementary Figure S4G). (O, P) CFZ $(1 \mu \mathrm{M})$ induces CD11b $(0)$ and CD61 $(\mathrm{P})$ in CD34 ${ }^{+} \mathrm{CP}-\mathrm{CML}$ cells (histograms in Online Supplementary Figure S4H). Graphs illustrate the mean \pm standard error mean. $* P<0.05$, $* * P<0.01$, $* * * P<0.001$; one-way analysis of variance followed by the Bonferroni post-test (except J, 0 , P; unpaired two-tailed t-test, $\mathrm{H}$; Kruskal-Wallis test followed by the Dunn test, M; left panel, as indicated and right panel; Mann-Whitney U test). *Vehicle vs. treatment, "imatinib vs. other treatments, ${ }^{\$}$ dasatinib vs. CFZ. Microscopic images; $n=3$ (Leica DMI6000B, 30 fields/group). Blots are representative of three independent experiments (all full blots in Online Supplementary Figure S14). A letter P followed by a number (in N and subsequent figures) designates the patient's identity. PARP: poly (ADP-ribose) polymerase; Cyt C: cytochrome C; CC; cleaved caspase; V: vehicle; IMT; imatinib; Dasa; dasatinib; FD; freshly diagnosed, Resp; imatinib-responder, Res; imatinib-resistant. 
A

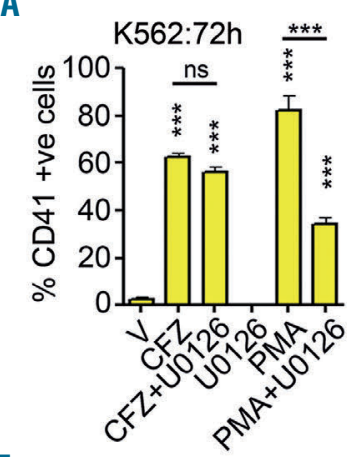

E

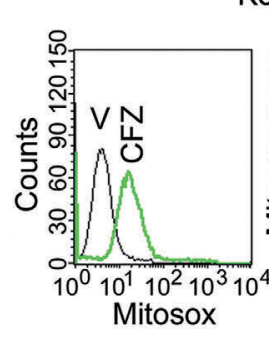

K562:24h
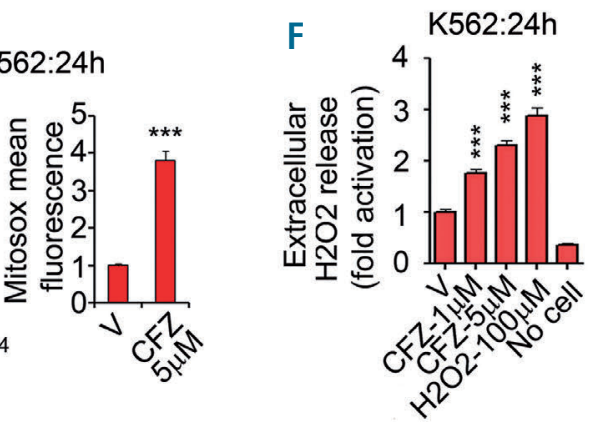

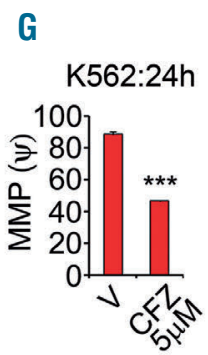

K562

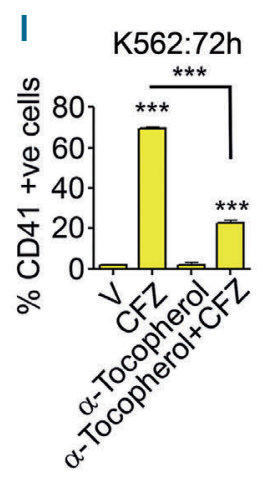

B

K562

CFZ-5 $\mu$ M PMA-10ng/ml (MW:

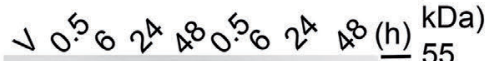

P-ERK1/2 - - - 55

ERK $1 / 2$ c

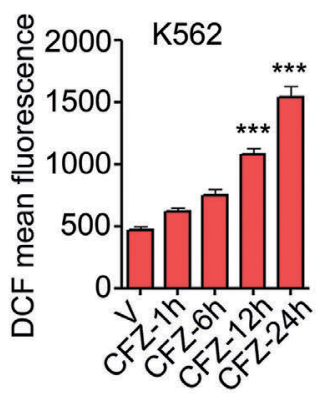

D

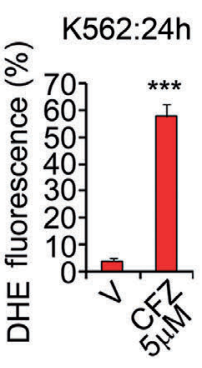

H
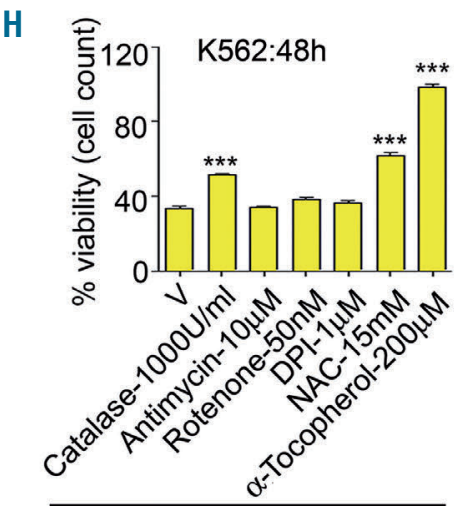

CFZ-6.5 $\mu \mathrm{M}+$

HEK: $24 \mathrm{~h}$

K

K562

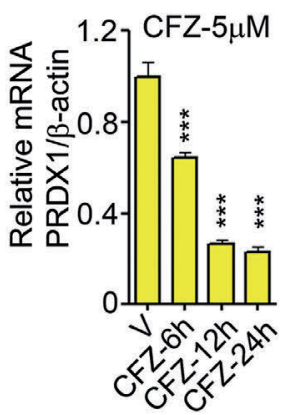

$\square \mathrm{V}$

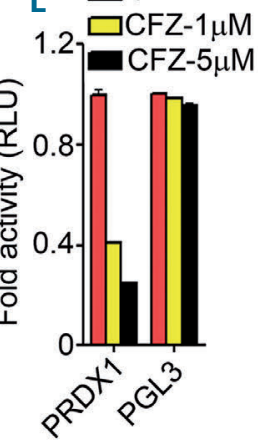

$\beta$-actin

M

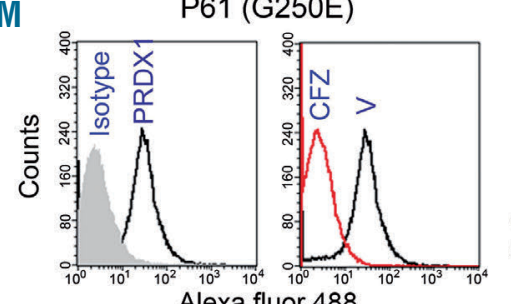

Relative PRDX1 (mean fluorescence)

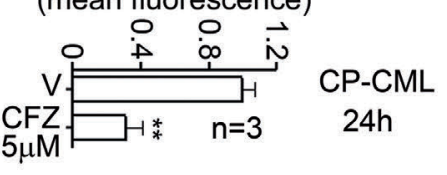

Figure 2. Clofazimine downregulates PRDX1 expression, which leads to reactive oxygen species-dependent differentiation and apoptosis. (A) Phorbol myristate acetate $(10 \mathrm{ng} / \mathrm{mL}$ ) but not clofazimine (CFZ) $(2.5 \mu \mathrm{M})$-induced CD41 expression in K562 cells is blocked by U0126 (10 uM; $30 \mathrm{~min}$ pre-treatment). Representative histograms are shown in Online Supplementary Figure S5A. (B) CFZ does not induce ERK phosphorylation. (C-F) CFZ induces the production of cellular reactive oxygen species (ROS). Total ROS (C; representative histograms in Online Supplementary Figure $S 5 B)$, total superoxide (D), mitochondrial superoxide (E), and $\mathrm{H}_{2} \mathrm{O}_{2}(\mathrm{~F})$. (G) CFZ induces mitochondrial membrane depolarization (dot plot in Online Supplementary Figure S5C). (H) $\alpha$-tocopherol completely blocks CFZ-induced loss of K562 viability. (I) $\alpha$-tocopherol $(200 \mu \mathrm{M})$ blocks CFZ (2.5 $\mu \mathrm{M})$-induced CD41 expression in K562 cells (representative histograms in Online Supplementary Figure S5D). (J) CFZ reduces peroxiredoxin 1 (PRDX1) protein level within $12 \mathrm{~h}$. (K) CFZ reduces PRDX1 mRNA within $6 \mathrm{~h}$ in K562 cells. (L) CFZ reduces a PRDX1 (-1096+83 ) promoter-driven luciferase reporter activity in HEK-293 cells. (M) CFZ reduces PRDX1 protein in cells from patients with imatinib-resistant chronic phase chronic myeloid leukemia. Immunoblots are representative of three independent experiments. Graphs illustrate the mean \pm standard error of mean. $* * P<0.01, * * * P<0.001$ (A,C,F,H,I,K; one-way analysis of variance followed by the Bonferroni post-test. D,E,G,M; unpaired two-tailed Student $t$-test). V: vehicle; PMA: phorbol myristate acetate, ERK: extracellular signal-regulated kinase; MW: molecular weight; DHE: dihydroethidium; MMP: matrix metalloproteinase; DPI: diphenyleneiodonium; NAC: N-acetylL-cysteine; SOD: superoxide dismutase; NFE2L2: Nuclear factor erythroid 2 like 2; CP-CML: chronic phase chronic myeloid leukemia. 
clofazimine altered the expression of factors that modulate cellular ROS or impart protection against them. Evaluation of peroxiredoxin thioperoxidases, which catalyze reduction of peroxynitrite, $\mathrm{H}_{2} \mathrm{O}_{2}$ and organic hydroperoxides ${ }^{24,28}$ revealed that clofazimine reduced PRDX1 expression at $12 \mathrm{~h}$ (Figure 2J) which coincided with clofazimine-induced ROS production (Figure 2C). Clofazimine also reduced PRDX3 (24 h), PRDX2 and PRDX5 (48 h) but not PRDX4 and PRDX6 (Figure 2J). Clofazimine suppressed cytosolic superoxide dismutase SOD1 (24 h onwards) but not mitochondrial SOD2 expression (Figure 2J). Nuclear factor erythroid 2 like 2 (NFE2L2), which regulates expression of various cytoprotective and multidrug resistant proteins ${ }^{29}$ was also downregulated by clofazimine ( $24 \mathrm{~h}$ onwards; Figure 2J). Clofazimine did not alter catalase expression (Figure 2J).

Since suppression of PRDX1 expression was the most proximal event observed (Figure 2J), we studied it in detail. Clofazimine suppressed PRDX1 mRNA in K562 cells as early as $6 \mathrm{~h}$ (Figure $2 \mathrm{~K}$; quantitative real-time polymerase chain reaction primer sequences are listed in Online Supplementary Table S2) indicating that clofazimine may regulate the PRDX1 promoter. We thus assessed clofazimine's effect in HEK-293 cells transfected with a PRDX1 promoter-driven luciferase reporter (PRDX1-luc; 1065-+83) or an empty reporter and found that clofazimine specifically repressed the PRDX1-luc (Figure 2L), confirming that it modulates the PRDX1 promoter. Figure $2 \mathrm{~L}$ also indicates that factor(s) responsible for clofaziminemediated downregulation of the PRDX1 promoter is(are) endogenously expressed in HEK-293. Clofazimine also reduced PRDX1 protein in CML cells (Figure $2 \mathrm{M}$ ).

\section{Introduction of exogenous PRDX1 ameliorates clofazimine-induced generation of cellular reactive oxygen species, differentiation and apoptosis}

We next asked whether clofazimine's actions were mediated by PRDX1 and thus conducted rescue experiments with exogenous PRDX1. PRDX1 overexpression in K562 cells abrogated clofazimine-induced ROS production (Figure $3 \mathrm{~A}$ ), caspase cleavage and BAX expression (Figure 3B). Clofazimine-induced K562 apoptosis and differentiation were also blocked in PRDX1-transfected cells (Figure 3C, D and Online Supplementary Figure S6A,B). Consistently, clofazimine failed to induce ROS and apoptosis in PRDX1transfected CML CD34+ cells (Figure 3E-H). Given its observed protective function we investigated whether PRDX1 expression is elevated in CML cells and found that there was a trend to increased PRDX1 mRNA (albeit statistically insignificant) in CP-CML cells compared to healthy donor cells (Figure 3I). We also compared PRDX1 levels in LSC versus non-LSC. Both CD34+38+ and CD34+38 cells expressed significantly higher levels of PRDX1 transcript than did CD34-38 cells (which were primarily gated monocytic cells), with the highest expression observed in the CD $34^{+} 38^{+}$population (Figure 3J, Online Supplementary Figure S6C). These results indicate that clofazimine-mediated transcriptional repression of PRDX1 expression plays a key role in imparting clofazimine's actions.

\section{Clofazimine-mediated suppression of PRDX1 expression is achieved through downregulation of MYB expression}

Clofazimine decreased PRDX1 mRNA at $6 \mathrm{~h}$ (Figure $2 \mathrm{~K}$ ) and suppressed a PRDX1 promoter reporter (Figure
2L), indicating that it may regulate transcription factors that modulate the PRDX1 promoter. A literature search revealed predicted binding sites for MYB, E2F transcription factor 1 (E2F1), glucocorticoid receptor (GR), CCAAT/enhancer binding protein alpha (CEBP $\alpha$ ), cAMP response element binding protein (CREB), activating transcription factor 4 (ATF-4) and activator protein 1 (AP-1) on the PRDX1 promoter. $^{30}$ In K562 cells, clofazimine treatment decreased MYB expression from $3 \mathrm{~h}$ and CREB from $12 \mathrm{~h}$ onwards but had only a modest or no effect on GR, E2F1, C-Jun, C-Fos and ATF-4 (Figure 4A, Online Supplementary Figure S7).

Since MYB downregulation was the most proximal event observed which preceded PRDX1 downregulation and that MYB is endogenously expressed in HEK-293 cells, ${ }^{31}$ we studied it in detail. Clofazimine reduced $M Y B$ transcripts in K562 cells from $1 \mathrm{~h}$ onwards (Figure 4B). Consistently, clofazimine suppressed a canonical MYB response element-driven reporter ( $3 X \mathrm{MRE}$ ), while transfection of exogenous MYB rescued it (Figure 4C) We next assessed whether MYB could regulate PRDX1 promoter, and introduction of exogenous MYB did indeed activate PRDX1-luc (-1065-+83) (Figure 4D). Furthermore, clofazimine downregulated PRDX1-luc in vector-transfected cells, and introduction of exogenous MYB dampened it (Figure 4D). These results indicate that MYB regulated the PRDX1 promoter probably by directly binding to it, and clofazimine suppressed PRDX1 expression by downregulating endogenous MYB expression. We thus attempted to identify the MYBresponsive element on the PRDX1 promoter. PRDX1 promoter deletion-mapping revealed that MYB activated the $-11-+83$ but not the $+9-+83$ construct (Figure 4E). Analysis of the $-11-+9$ region revealed a sequence resembling the consensus MYB binding site "PyAACG/TG" $/ 32,33$ in reverse and complementary orientation; "CCGTTC", at position -8--3. Mutation of this sequence in the $-11-+83$ promoter reporter to "CCGggC", led to complete loss of MYB-responsiveness (Figure $4 \mathrm{~F}$ ). To further confirm that this sequence is indeed the MYB-responsive region on the PRDX1 promoter, we constructed a reporter containing three copies of the $-11-+9$ sequence (PRDX-MYB-RE) and co-transfected it with MYB or empty vector and found that MYB did indeed specifically activate PRDX-MYB-RE (Figure 4G). Chromatin immunoprecipitation confirmed that MYB was recruited on the PRDX1 promoter and that clofazimine reduced its recruitment, and consequently reduced histone $\mathrm{H} 3$ acetylation (indicating reduced transcription) (Figure 4H). Consistently, clofazimine reduced MYB protein in CP-CML (Figure 4I-J), and MYB transcript in CML CD34+ cells (Figure 4K).

We next assessed whether the introduction of exogenous MYB could compromise clofazimine's actions and found that MYB overexpression in K562 cells did indeed mitigate the clofazimine-mediated decrease in PRDX1 expression and increase in caspase-3 cleavage, apoptosis, differentiation and ROS (Figure 4L-O, Online Supplementary Figure $S 8 A, B)$. MYB mRNA expression in both imatinib-resistant and -responsive CP-CML cells was significantly higher than in control cells (Figure $4 \mathrm{P}$ ). These results indicate that MYB binds to the PRDX1 promoter and regulates its expression and clofaziminemediated cellular functions are achieved through downregulation of MYB. 
Clofazimine reduces MYB expression by rapid degradation of p65 NFKB protein

Clofazimine reduced MYB mRNA expression from $1 \mathrm{~h}$ in K562 cells (Figure 4B); we therefore investigated whether it regulates any factor that regulates MYB expression itself. A literature search revealed that NFKB transcription factors regulate MYB expression by various mechanisms. ${ }^{34-37}$ Consistent with a reported NFKB response element (NFKB-RE) in the MYB promoter situated at -278 to $-256 \mathrm{bp}$ upstream of the transcriptional start site $^{37}$ tumor necrosis factor- $\alpha$ treatment, or p65/RELA transfection activated a $M Y B(-687-+204)$ promoter reporter $^{38}$ (Figure 5A). Given that clofazimine downregulated PRDX 1 promoter luc in HEK-293 cells (Figure 2L) and that p65 is endogenously expressed in HEK-293 cells, ${ }^{39}$ we investigated whether clofazimine regulates p65 expression. Clofazimine reduced p65 protein in K562 cells within 15 min, without affecting other NFKB family members, p50, p105 and C-Rel (Figure 5B). Clofazimine did, however, fail to alter p 65 mRNA expression (Figure 5C) indicating that clofazimine-mediated p65 downregulation may happen at a post-transcriptional or -translational level.
A

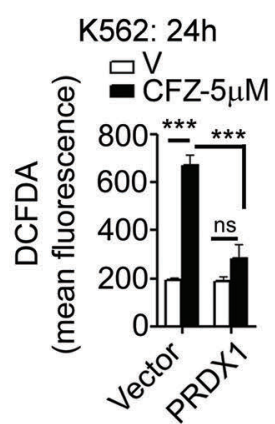

B

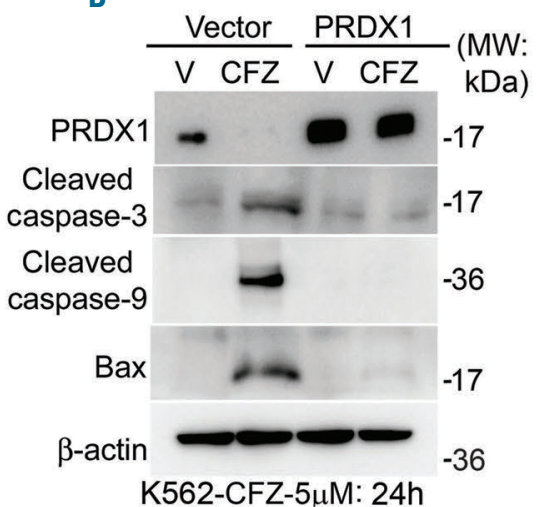

C

K562:48h 믈 $\mathrm{CFZ}-5 \mu \mathrm{M}$

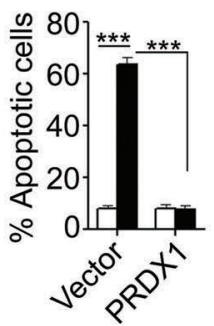

D

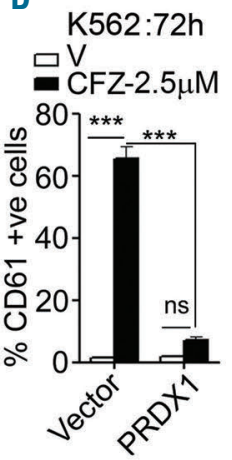

$\mathrm{E}$

CD34+ cells : $24 \mathrm{~h}$

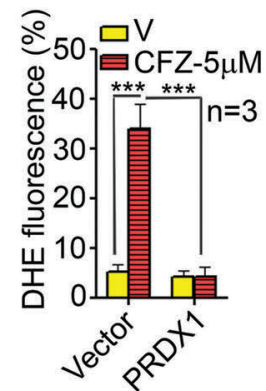

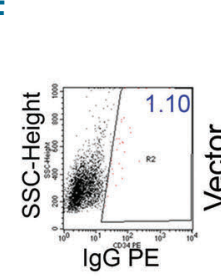
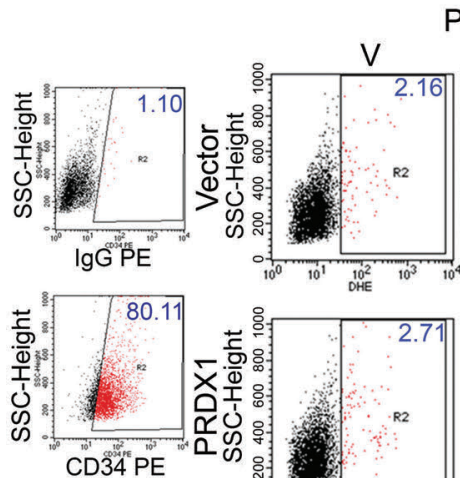

P81
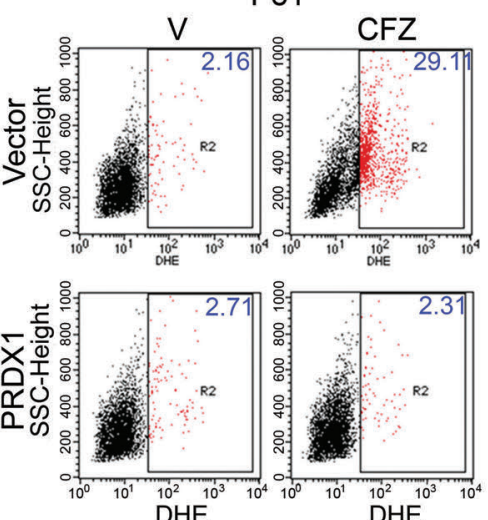

Relative mRNA

PRDX1/GAPDH

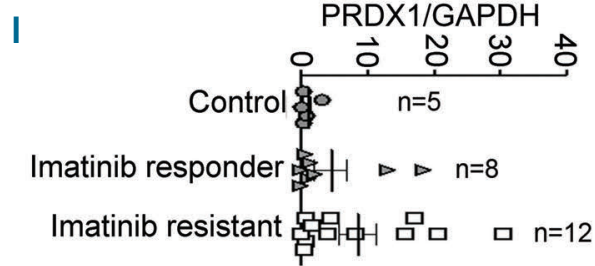

G $\square \mathrm{V}$ 回CFZ-5 $\mu \mathrm{M}$ CD34+ cells $48 \mathrm{~h}$
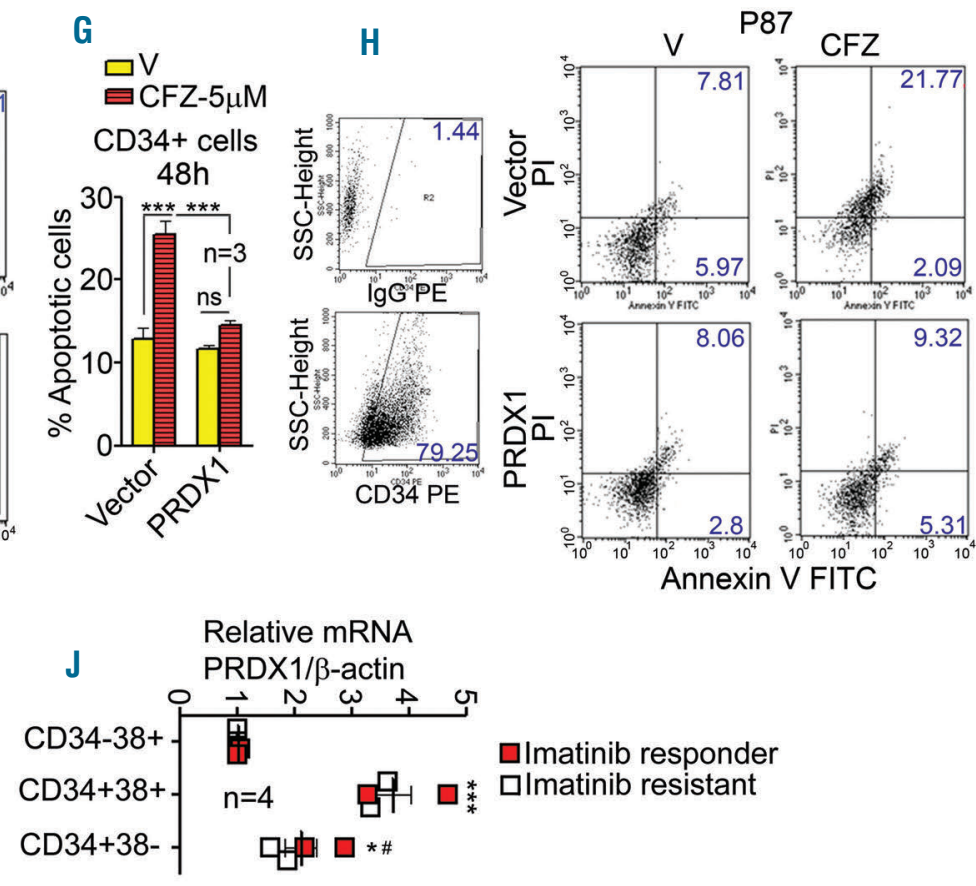

Figure 3. Introduction of exogenous PRDX1 in cells compromises clofazimine-induced reactive oxygen species generation, apoptosis and differentiation. (A-D) Overexpression of peroxiredoxin 1 (PRDX1) in K562 cells ameliorates clofazimine (CFZ)-induced reactive oxygen species generation (A), caspase cleavage and BAX expression (B), apoptosis (C; representative dot plots in Online Supplementary Figure S6A), and CD61 expression (D; representative histograms in Online Supplementary Figure S6B). (E-H). CD34 ${ }^{+}$chronic myeloid leukemia (CML) cells transfected with PRDX1 are protected from CFZ-induced ROS generation and apoptosis. $(\mathrm{E}, \mathrm{F}) \mathrm{CD} 34^{+}$cells were isolated from chronic phase (CP)-CML cells as described above and were transfected with empty vector or a PRDX1 expression plasmid. Cells were then treated with CFZ $(5 \mu \mathrm{M} ; 24 \mathrm{~h})$ and dihydroethidium fluorescence was measured by flow cytometry (E; graphical representation, F; representative dot plots). (G, H) CD34+ cells transfected with empty vector or PRDX1 were treated with CFZ $(5 \mu \mathrm{M} ; 48 \mathrm{~h})$ and apoptosis was assessed by annexin $V$ staining followed by flow cytometry (G; graphical representation, $\mathrm{H}$; representative dot plots). (I) PRDX1 mRNA expression in CP-CML cells determined by quantitative real-time polymerase chain reaction (QRT-PCR). (J) PRDX1 mRNA expression in CD34 $38^{+}, \mathrm{CD}_{3} 4^{+} 38^{+}$and $\mathrm{CD} 34^{+} 38$ cells by QRT-PCR Graphs (except I \& J) are mean \pm standard error of mean of three independent experiments. Immunoblots are representative of three independent experiments. $* * P<0.01, * * * P<0.001$ (A,C,D-E,G; two-way analysis of variance followed by the Bonferroni post-test. I,J; Kruskal-Wallis test followed by the Dunn test). DCFDA: 2',7'-dichlorofluorescein diacetate; V: vehicle; SSC; side scatter; DHE: dihydroethidium; PE: phycoerythrin. 
Furthermore, consequent to its downregulation of p65, clofazimine suppressed an NFKB-RE reporter (Figure 5D). We next investigated whether the clofazimine-mediated rapid decrease in p65 was due to proteasomal degradation, and found that clofazimine failed to reduce p65 in the presence of the proteasomal inhibitors MG132 and lactacystin (Figure 5E). p65 degradation was associated with its increased ubiquitination by clofazimine (Figure $5 F)$. These results indicate that clofazimine causes p65 ubiquitination leading to its proteasomal degradation. Clofazimine also significantly decreased p65 protein in CP-CML cells (Figure 5G).
A

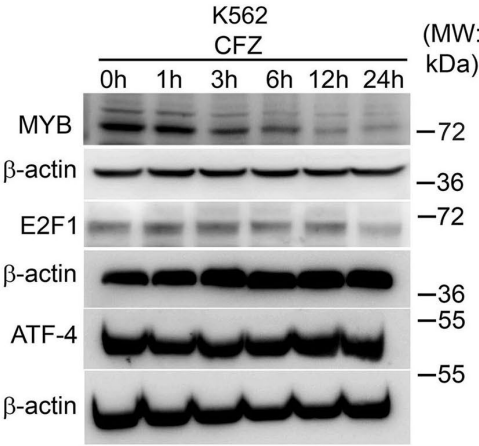

D

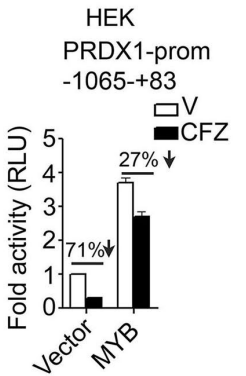

H

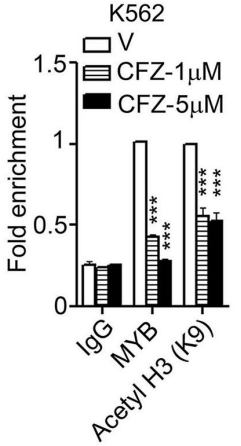

E

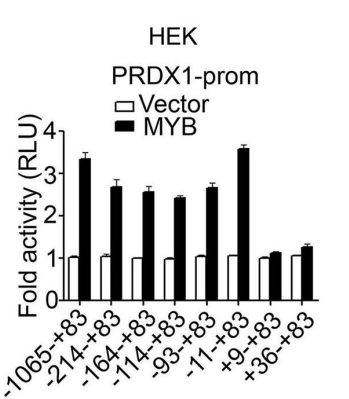

B

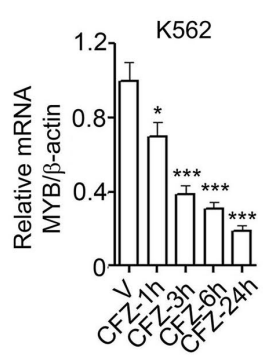

F

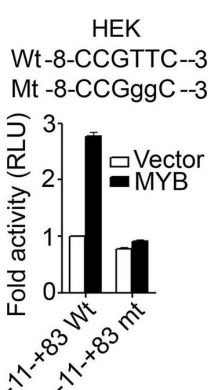

J

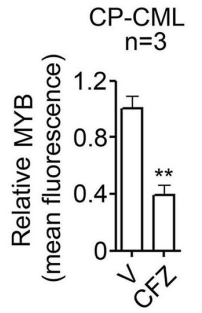

C

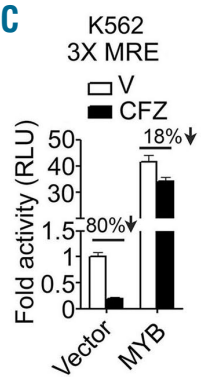

G

$$
\begin{gathered}
\text { HEK } \\
\text { Vector } \\
\text { GYB }
\end{gathered}
$$
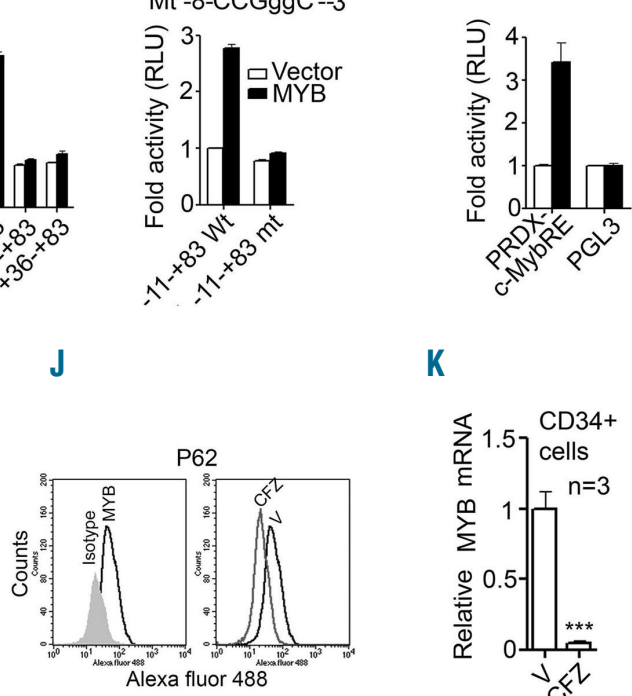

K

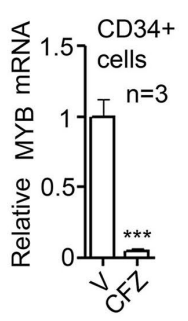

$\mathbf{L}$

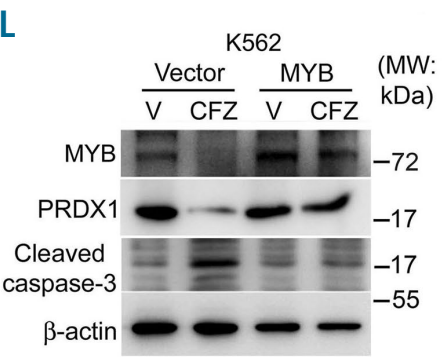

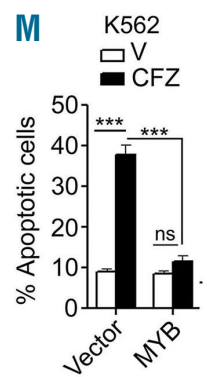

N

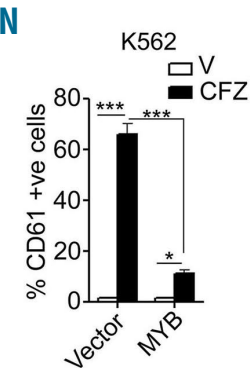

0

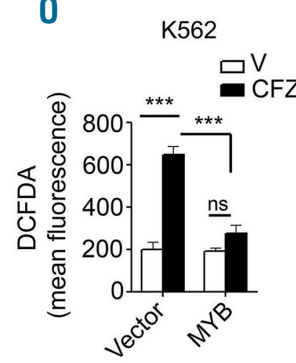

Figure 4. Clofazimine modulates PRXD1 expression via transcriptional regulation of MYB. (A, B) Clofazimine (CFZ) $(5 \mu \mathrm{M})$ reduces myeloblastoma oncoprotein (MYB) protein (A), mRNA (B) expression in K562 cells. (C) CFZ (5 $4 \mathrm{M}, 24$ h) reduces a three copy MYB consensus response element-containing reporter activity in K562 cells and overexpression of MYB, mitigates this repression. (D) CFZ (5 $\mu \mathrm{M}, 24$ h) represses the peroxiredoxin 1 (PRDX1) promoter-luc and overexpression of MYB activates this reporter and ameliorates its CFZmediated repression. (E) Deletion mapping of the $\sim 1 \mathrm{~kb}$ PRDX1 promoter in HEK-293 cells reveals MYB response between the -11 to +9 region in the MYB promoter. $(F)$ MYB activates a reporter containing -11 to +83 wildtype (Wt) but not mutated ( $\mathrm{mt}$; mutated bases in lower case letters) PRDX1 promoter sequence in HEK-293 cells. (G) MYB activates a reporter containing the -11 to +9 PRDX1 promoter sequence in three tandem repeats in HEK-293 cells. (H) MYB is recruited to the endogenous PRDX1 promoter in K562 cells and CFZ (24 h) reduces its recruitment and concomitantly reduces histone 3 (K9) acetylation. (I, J) CFZ (5 $\mu \mathrm{M}$, $24 \mathrm{~h}$ ) reduces MYB protein in imatinib-resistant chronic phase chronic myeloid leukemia (CP-CML) cells (I; graphical representation, J; representative histogram). (K). CFZ (5 $\mu \mathrm{M}, 24 \mathrm{~h}$ ) suppresses MYB expression in $\mathrm{CD}_{3} 4^{+}$cells. (L-O; treatment concentration and duration same as Figure 3A-D) Overexpression of MYB in K562 cells mitigates CFZmediated downregulation of PRDX1 protein level, and induction of caspase-3 cleavage (L), enhanced K562 apoptosis (M; dot plots in Online Supplementary Figure S8A), CD61 expression (N; histograms in Online Supplementary Figure S8B) and cellular reactive oxygen species (O). (P) MYB mRNA expression in CP-CML cells. Graphs, except (P), show the mean \pm standard error of mean of three independent experiments. Immunoblots are one representative of three independent experiments. $* P<0.05, * * P<0.01$, $* * * P<0.001$. (B, H) one-way, (M-O) two-way ANOVA followed by the Bonferroni post-test. (I, K); unpaired two-tailed Student $t$-test. (P) Kruskal-Wallis test followed by the Dunn test. E2F1; E2F transcription factor ATF-4: activating transcription factor 4; RLU: relative light unit; 3X MRE: three copy MYB consensus response element; $V$; vehicle; DCFDA: 2',7'-dichlorofluorescein diacetate; GAPDH: glyceraldehyde 3-phosphate dehydrogenase. 
We next evaluated whether the introduction of exogenous p65 could affect clofazimine's actions and found that p65 overexpression did indeed ameliorate the clofazimine-mediated decrease in MYB and PRDX1 expression, increased caspase- 3 cleavage (Figure $5 \mathrm{H}$ ), apoptosis, differ- entiation and ROS production in K562 cells (Figure 5I-K, Online Supplementary Figure S9A, B). There was a trend (albeit not statistically significant) to increased p65 mRNA in CP-CML cells compared to the level in cells from healthy donors (Figure 5L). Together, the results indicate
A

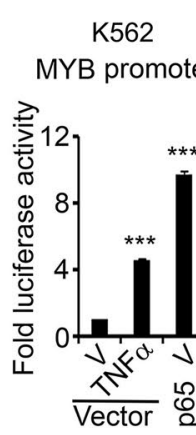

B

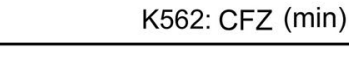

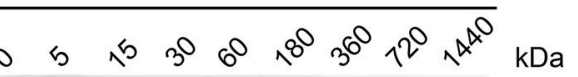
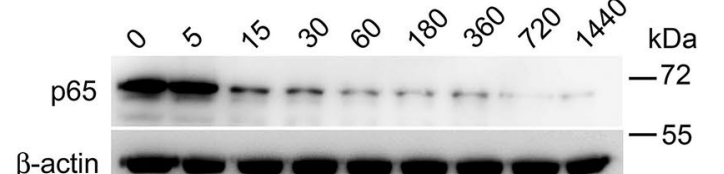

p105 - - - - - - - - 95

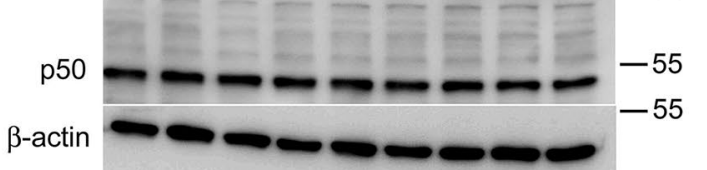

C-Rel

C-Re

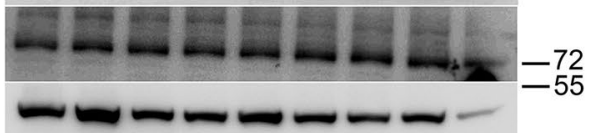

C

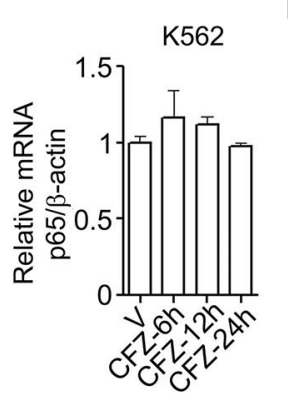

D

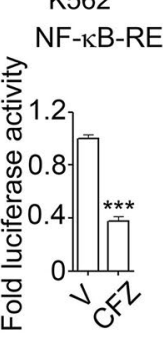

E

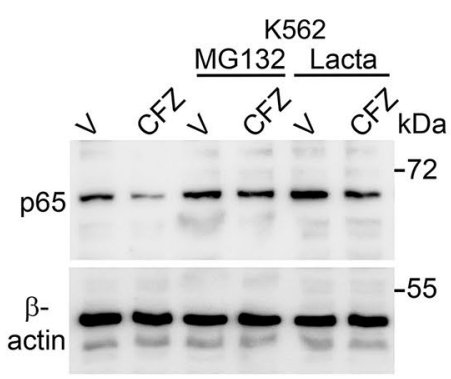

F

K562

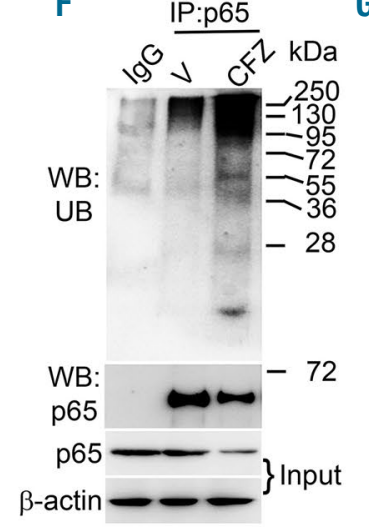

G

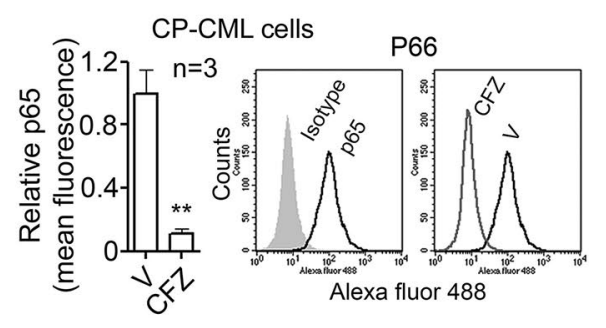

H
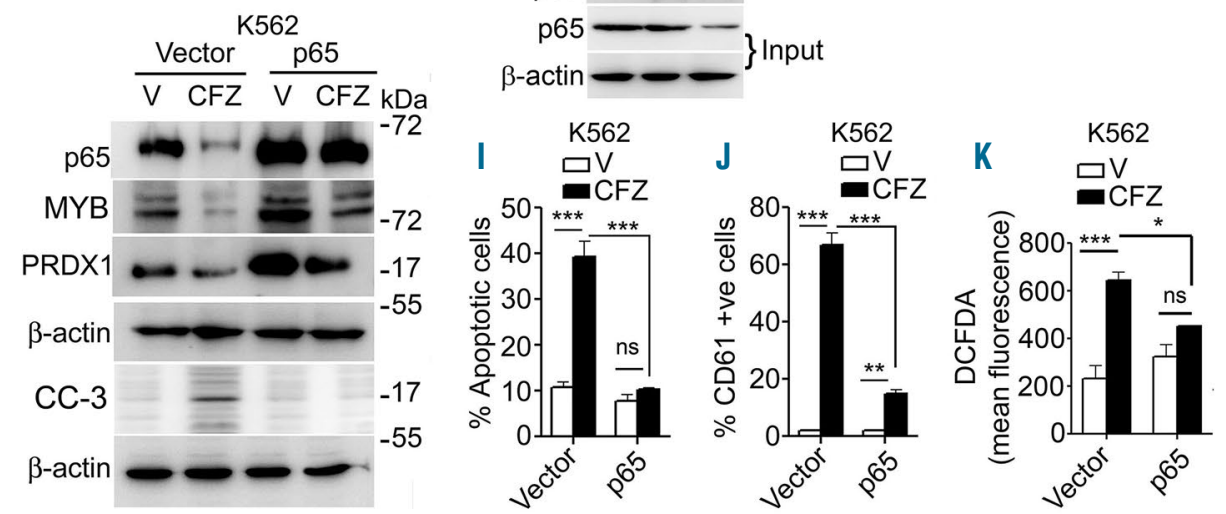

$\mathrm{L}$

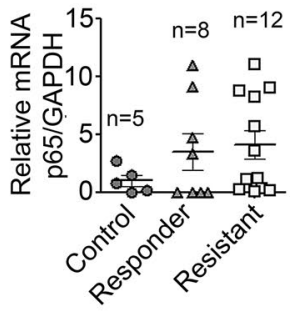

Figure 5. Clofazimine regulates myeloblastoma oncoprotein expression via rapid proteasomal degradation of p65 NFKB. (A) Transfected p65 or tumor necrosis factor- $\alpha(10 \mathrm{ng} / \mathrm{mL})$ activates a myeloblastoma oncoprotein (MYB) promoter (-687-+204)-driven luciferase reporter in K562 cells. (B, C) Clofazimine (CFZ) (5 $\mu \mathrm{M})$ rapidly reduces p65 protein level (B) but does not alter p65 mRNA (C) in K562 cells. (D) CFZ (5 $\mu \mathrm{M}, 24 \mathrm{~h})$ reduces a nuclear factor kappa B response element-driven reporter in K562 cells. (E) MG132 or lactacystin ( $10 \mu \mathrm{M}, 6 \mathrm{~h}$ pretreatment) prevents the CFZ ( $5 \mu \mathrm{M}, 1 \mathrm{~h}$ )-mediated reduction in p65 protein level in K562 cells. (F) CFZ (5 $\mu \mathrm{M}, 1 \mathrm{~h})$ induces ubiquitination of p65. Proteins were immunoprecipitated with a rabbit p65 antibody followed by western blotting with mouse ubiquitin or p65 antibodies. To avoid the possibility of detection of IgG heavy chain ( $\sim 50 \mathrm{kDa})$, an IgG light chain-specific secondary antibody was used. (G) CFZ (5 $\mu \mathrm{M}, 24 \mathrm{~h})$ reduces p65 protein in imatinib-resistant chronic phase chronic myeloid leukemia (CP-CML) cells. (H-K; treatment concentration and duration same as in Figure $3 \mathrm{~A}-\mathrm{D})$ p65 overexpression in K562 cells mitigates CFZ-induced downregulation of MYB and peroxiredoxin 1 and upregulation of caspase 3 cleavage (H), CFZ-mediated apoptosis (I; dot plots in Online Supplementary Figure S9A), CD61 expression (J; dot plots in Online Supplementary Figure S9B) and generation of cellular reactive oxygen species (K). (L) p65 expression in CP-CML cells. Graphs (except L) illustrate the mean \pm standard error of mean of three independent experiments. (B,E,H) are one representative of three independent experiments. $(\mathrm{F})$ is one representative of two independent experiments. $* P<0.05$, $* * P<0.01$, $* * * P<0.001$. (A) One-way analysis of variance (ANOVA), (I-K) Two-way ANOVA followed by the Bonferroni post-test. (C, L); Kruskal-Wallis test followed by the Dunn test. (D, G) Unpaired two-tailed Student $t$-test. MYB: myeloblastoma oncoprotein; V: vehicle; NFKB: uclear factor kappa B; TNF- $\alpha$ : tumor necrosis factor-alpha; Lacta: lactacystin; IP: immunoprecipitation; WB: western blot; UB: ubiquitin; PRDX1: peroxiredoxin 1; CC; cleaved caspase; DCFDA: 2',7'-dichlorofluorescein diacetate; GAPDH: glyceraldehyde 3-phosphate dehydrogenase. 
A

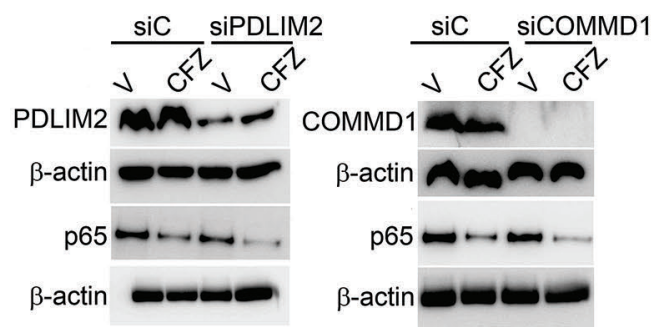

K562

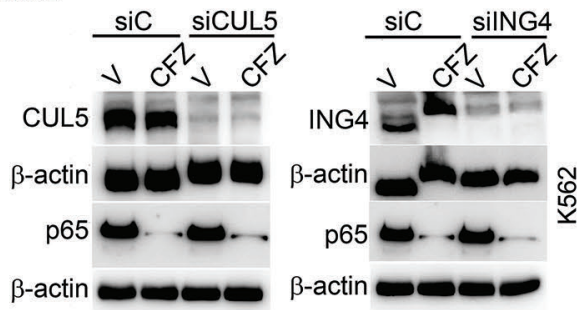

B

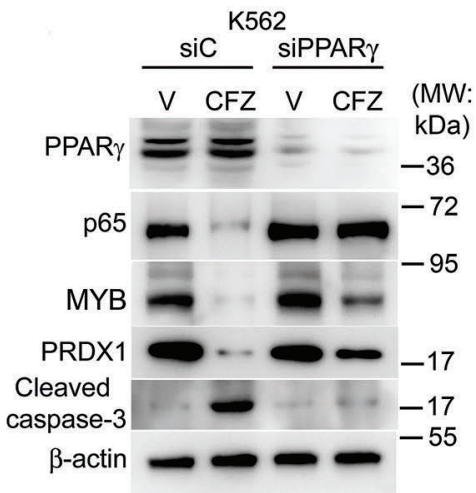

C

K562 D

E K562

믄 $\quad$ GFZ
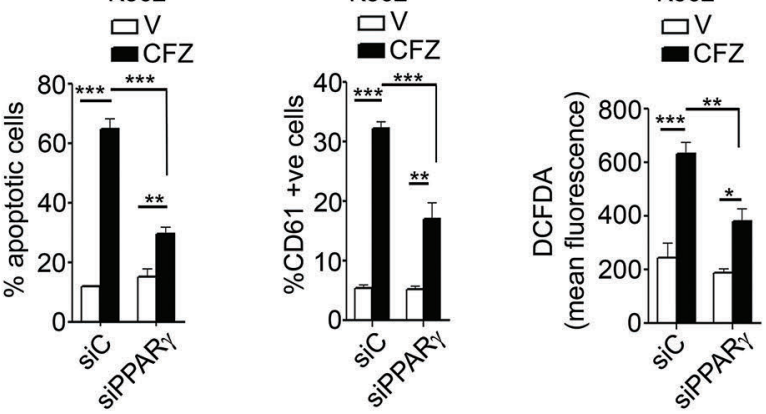

$\mathrm{H}$

Time (min)
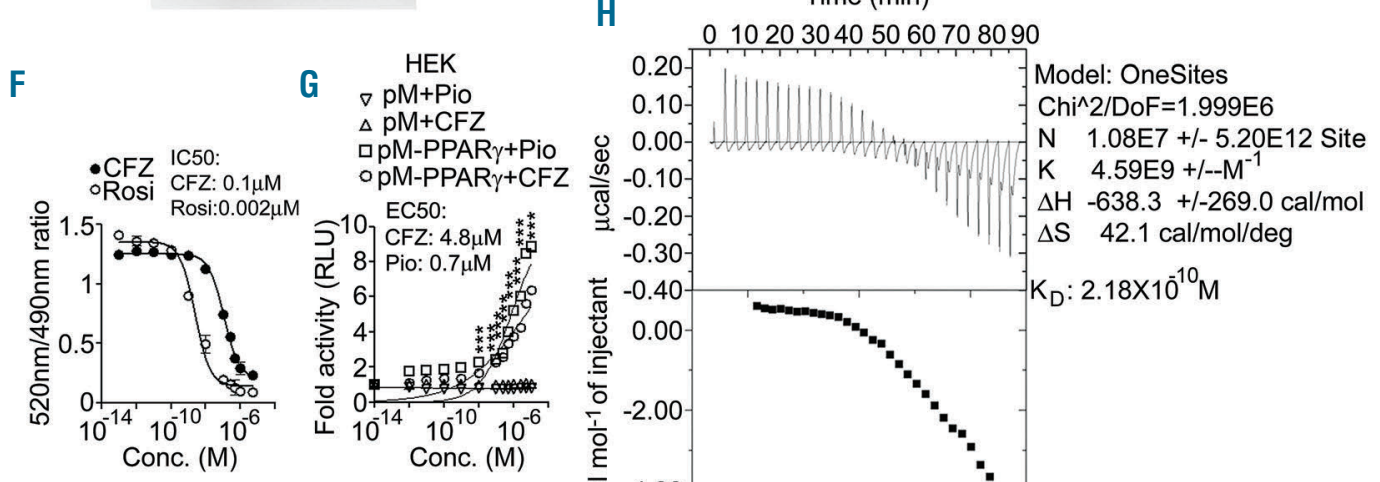

-0.20
-0.30

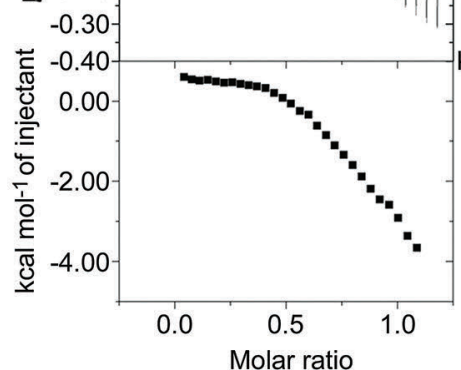

I
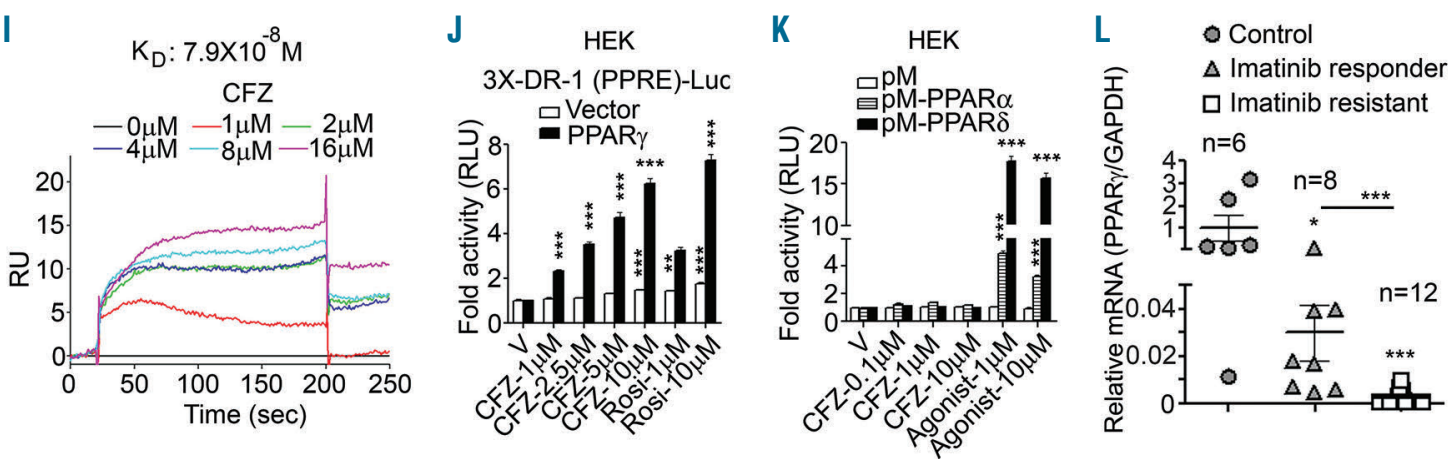

Figure 6. Clofazimine regulates p65 level, apoptosis and differentiation via a direct interaction with PPAR . (A) Depletion of ubiquitin ligases PDLIM2, COMMD1, Cul5 and ING4 does not affect clofazimine (CFZ) $(5 \mathrm{uM}, 24 \mathrm{~h}$ )-mediated decrease in p65 protein (B-E). Treatment concentration and duration same as in Figure $3 \mathrm{~A}-$ D). Peroxisome proliferator-activated receptor (PPAR)- $\gamma$ depletion in K562 cells compromises CFZ-induced downregulation of p65, myeloblastoma oncoprotein (MYB), and peroxiredoxin 1 (PRDX1) and upregulation of cleaved caspase-3 (B), apoptosis (C; dot plots in Online Supplementary Figure S10A), CD61 expression (D; histograms in Online Supplementary Figure S10B) and generation of reactive oxygen species (E). (F-J) CFZ physically interacts with PPAR and increases its transcriptional activity. (F) The PPAR $\gamma$-CFZ interaction as determined by a cell-free time-resolved fluorescence resonance energy transfer lanthascreen assay. (G) CFZ increases transcriptional activity of a Gal4DBD-PPAR $y$ LBD fusion protein (pM-PPAR $\gamma$ ) on a GAL4 response element-containing reporter (GAL4-UAS-Luc) in transfected HEK-293 cells $(\mathrm{H})$ CFZ alters thermodynamic properties of purified PPAR $\mathrm{LBD}$. Isothermal titration calorimetry to probe interaction of CFZ with PPAR $\gamma \mathrm{LBD}$ (protein purification and characterization in Online Supplementary Figure S11A-D); CFZ $(250 \mu \mathrm{M})$ was titrated into PPAR $\mathrm{LBD}$ solution $(50 \mu \mathrm{M})$. The titration curve shows a series of endothermic reactions followed by exothermic isotherms. (I) The interaction between PPAR $\gamma$-LBD and CFZ was evaluated using a Biacore 3000 instrument. SPR sensogram curves showing the interactions between the indicated concentrations of CFZ and his-tagged PPARyLBD captured over an anti-his antibody immobilized CM5 chip. (J) CFZ increases three-copy DR-1 PPRE luc reporter activity in HEK-293 cells transfected with a PPAR expression plasmid. (K) CFZ does not alter transcriptional

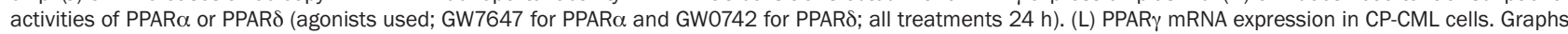
(except $\mathrm{L}$ ) are mean \pm SEM of three independent experiments. Blots are one representative of three independent experiments. ( $\mathrm{H}-\mathrm{I})$ One representative of two independent experiments. $* P<0.05, * * P<0.01, * * * P<0.001$ (C-E, G). Two-wayanalysis of variance (ANOVA), (J-K) One-way ANOVA followed by the Bonferroni post-test. L; (K) Kruskal-Wallis test followed by the Dunn test. 
that clofazimine causes $p 65$ proteasomal degradation by ubiquitinating it, which leads to sequential MYB and PRDX1 downregulation, ultimately resulting in the cellular effects imparted by clofazimine.

\section{Clofazimine functions through a direct interaction with PPAR $\gamma$.}

Since clofazimine induced p65 ubiquitination (Figure $5 \mathrm{~F})$, we next investigated whether clofazimine modulated any of the E3 ubiquitin ligases that are reported to ubiquitinate p65. PDZ and LIM domain protein 2 (PDLIM2), inhibitor of growth family member 4 (ING4), cullin 5 (CUL5), copper metabolism domain containing 1 (COMMD1; also called MURR1) and PPAR $\gamma$ ubiquitinate p65 and cause its proteasomal degradation. ${ }^{40,41}$ We thus assessed whether clofazimine acted through any of these factors. While RNAi-mediated depletion of PDLIM2, ING4, CUL5 and COMMD1 failed to affect clofazimineinduced p65 degradation (Figure 6A), PPAR $\gamma$ depletion mitigated clofazimine-mediated decrease in p65, MYB and PRDX1 and increase in caspase- 3 cleavage, apoptosis, differentiation and ROS (Figure 6B-E, Online Supplementary Figure $S 10 A, B$ ) indicating that clofazimine may modulate PPAR $\gamma$ activity (it is important to note here that HEK-293 cells also express endogenous PPAR $\gamma^{42,43}$ ).

We therefore evaluated whether clofazimine interacts with PPAR $\gamma$ and assessed its interaction with purified PPAR $\gamma$ protein in a cell-free, time-resolved fluorescence resonance energy transfer assay. Clofazimine successfully competed with a fluorophore-labeled PPAR $\gamma$ agonist for binding to purified PPAR $\gamma$-LBD with an $\mathrm{IC}_{50}$ of $0.1 \mu \mathrm{M}$ (Figure 6F). Since PPAR $\gamma$ is also a transcription factor, we assessed whether clofazimine also modulated its transcriptional activity in a heterologous system, in which the cells were transfected with pM-PPAR $\gamma$ [Gal4-DNA-binding-domain (DBD) fused to PPAR $\gamma$-ligand-binding domain] or an empty pM vector containing Gal4-DBD, and a Gal-UAS-luc reporter containing binding sites for GAL4-DBD. Clofazimine concentration-dependently increased the GAL-UAS reporter activity in the presence of pM-PPAR $\gamma$ but not pM alone (Figure 6G). To confirm clofazimine-mediated transcriptional activation of PPAR $\gamma$ we also studied it on a direct repeat-1 (DR-1; 3-copy) PPAR response element-driven reporter (PPRE-luc) using full-length PPAR $\gamma$ and, in this case too, clofazimine increased PPRE-Luc activity in the presence of transfected PPAR $\gamma$ (a modest response was also seen in vector-transfected cells; due to endogenous PPAR $\gamma$ ) (Figure 6J). To further probe the interaction of clofazimine with PPAR $\gamma$, we titrated clofazimine with PPAR $\gamma$-LBD (the purification and characterization of PPAR $\gamma-\mathrm{LBD}$ are illustrated in Online Supplementary Figure $S 11 A-D)$ by isothermal titration calorimetry. The titration curve of clofazimine with PPAR $\gamma$-LBD shows a series of endothermic reactions followed by exothermic isotherms (Figure $6 \mathrm{H}$ ). Stoichiometry calculated by integrating isotherms was one: i.e., one molecule of clofazimine bound to one macromolecule of PPAR $\gamma$-LBD. The equilibrium rate dissociation constant $\left(\mathrm{K}_{\mathrm{D}}\right)$ value was $0.2178 \mathrm{nM}$ (Figure $6 \mathrm{H}$ ). PPAR $\gamma$ also interacted with clofazimine in a surface plasmon resonance experiment (Figure 6I; the $\mathrm{K}_{\mathrm{D}}$ calculated by this method was $79 \mathrm{nM}$ ). Clofazimine did not alter the activities of $\mathrm{pM}$-PPAR $\alpha$ or $\mathrm{pM}-\mathrm{PPAR} \delta$, indicating its specificity for PPAR $\gamma$ (Figure 6K).

We next assessed whether there is any difference in
PPAR $\gamma$ mRNA expression between healthy control and CP-CML cells. A remarkably lower level of PPAR $\gamma$ transcripts was observed in the CP-CML cells than in healthy donors' cells, with the difference being more pronounced in cells from imatinib-resistant patients (Figure 6L). Together, these results demonstrate that clofazimine binds to PPAR $\gamma$ and modulates its transcriptional as well as E3 ubiquitin ligase activity and via its increased ubiquitin ligase activity PPAR $\gamma$ induces proteasomal degradation of p65 which in turn results in sequential transcriptional downregulation of $M Y B$ and PRDX1 leading to the cellular effects of clofazimine.

\section{Clofazimine shows superior cytotoxic activity compared to thiazolidinediones, acts in synergy with imatinib and drastically reduces quiescent CD34+ cells}

The PPAR $\gamma$ agonist pioglitazone synergizes with imatinib in eroding LSC by transcriptional downregulation but not dephosphorylation of STAT5. ${ }^{1}$ Since clofazimine modulated PPAR $\gamma$ transcriptional activity, we determined whether clofazimine also regulated STAT5 expression. As expected, clofazimine suppressed STAT5 protein (Figure 7A) and mRNA (Figure 7C) expression without altering its phosphorylation in K562 cells (Figure 7B). Consistent with the ability of PPAR $\gamma$ agonists to suppress BCL-2 expression in CML cells ${ }^{44}$ clofazimine decreased BCL-2 mRNA (Figure 7C) and protein (Figure 1D). Clofazimine did not alter CrkL or BCR-ABL1 phosphorylation (Figure 7D) indicating that it is not a BCR-ABL1 inhibitor per se. Furthermore, like pioglitazone, ${ }^{1}$ clofazimine also reduced $S T A T 5 B$ and other LSC maintenance factors such as HIF-1 $\alpha, H I F-2 \alpha$ and CITED2 transcripts in $\mathrm{CD}_{3} 4^{+}$cells from imatinibresistant patients (Figure $7 \mathrm{E}$ ).

We next compared the anti-CML efficacy of clofazimine with that of other PPAR $\gamma$ agonists. In a cell viability assay, clofazimine was found to be the most potent among all the PPAR $\gamma$ ligands tested. While the $\mathrm{IC}_{50}$ of clofazimine was $6.08 \mu \mathrm{M}$, those of rosiglitazone, troglitazone and pioglitazone were $32.28 \mu \mathrm{M}, 50.01 \mu \mathrm{M}$, and $37.39 \mu \mathrm{M}$, respectively (Figure $7 \mathrm{~F}$ ).

Since pioglitazone and imatinib synergistically inhibit CML cells, ${ }^{1}$ we assessed whether the same was true with clofazimine. In a K562 viability assay imatinib, dasatinib and clofazimine displayed $\mathrm{IC}_{50}$ values of $0.95 \mu \mathrm{M}, 0.64 \mu \mathrm{M}$ and $4.13 \mu \mathrm{M}$ respectively. However, combining $1.56 \mu \mathrm{M}$ clofazimine, which is close to the average human plasma level of clofazimine $(0.7 \mathrm{mg} / \mathrm{L})$ following daily oral administration of $100 \mathrm{mg}$ clofazimine, ${ }^{6}$ with imatinib reduced the $\mathrm{IC}_{50}$ of imatinib to $36.4 \mathrm{pM}$ (Figure $7 \mathrm{G}$ ). The combination index (CI) calculated using the Compusyn program revealed CI values $<1$ (Online Supplementary Table S3), indicating a synergistic effect. Compared to clofazimine, pioglitazone (48 h) showed a rather modest effect, which although synergistic (Online Supplementary Table S4), only reduced the $\mathrm{IC}_{50}$ of imatinib from $0.399 \mu \mathrm{M}$ (alone) to $0.052 \mu \mathrm{M}$ (with $5 \mu \mathrm{M}$ pioglitazone) or $0.032 \mu \mathrm{M}$ (with 10 $\mu \mathrm{M}$ pioglitazone) (Figure $7 \mathrm{H}$ ). Clofazimine also displayed synergism with dasatinib, where the $\mathrm{IC}_{50}$ of dasatinib of $0.64 \mu \mathrm{M}$ (alone) was reduced to $0.0124 \mu \mathrm{M}$ in the presence of clofazimine (Figure 7G) and the calculated CI was <1 (Online Supplementary Table S5). We next assessed whether the combination of imatinib and clofazimine, like pioglitazone, ${ }^{1}$ also eroded LSC. First, we performed a colonyforming assay in which clofazimine alone drastically reduced colony number compared to vehicle or imatinib, 
A

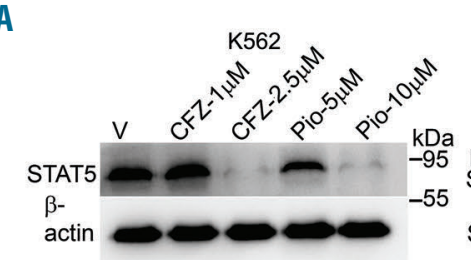

B

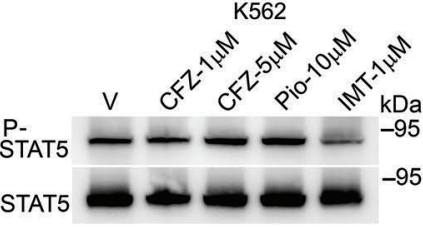

C

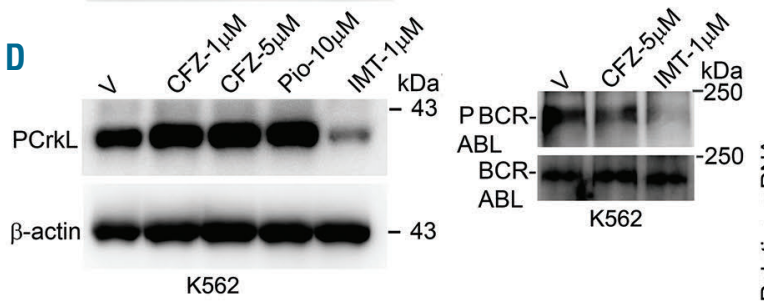

K562

\section{$E$}

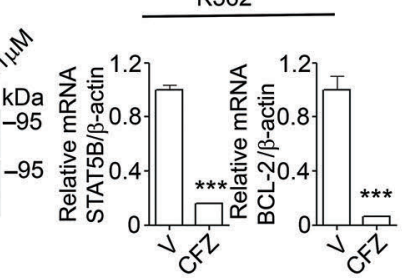

CD34+ cells $(n=3)$

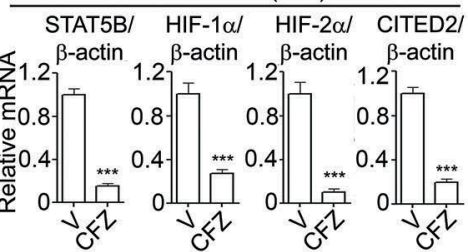

$\mathbf{F}$

G OCFZ

口Imatinib

$\nabla \mathrm{CFZ}-1.5 \mu \mathrm{M}+\mathrm{IMT}$

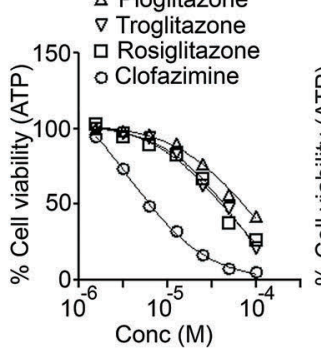

${ }^{120} \diamond \mathrm{CFZ}-1.5 \mu \mathrm{M}+\mathrm{Das}$

H

H oPioglitazone

$\square$ Imatinib

$\triangle \mathrm{Pio}-5 \mu \mathrm{M}+\mathrm{IMT}$

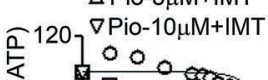

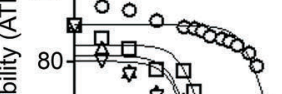

I

I CD34+ cells

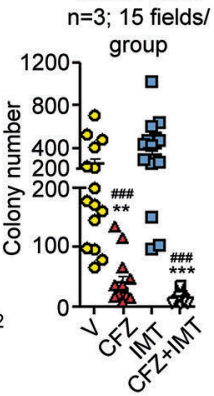

J

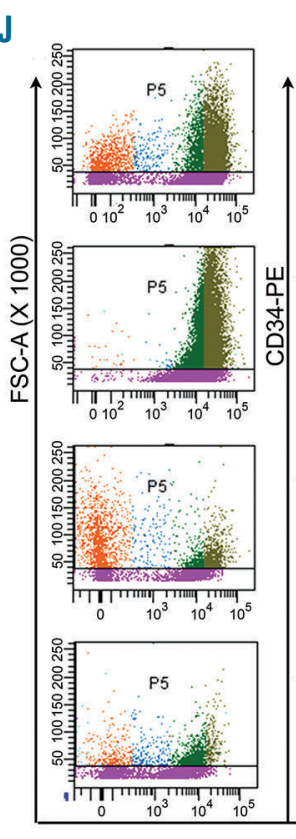

P78 (BC)
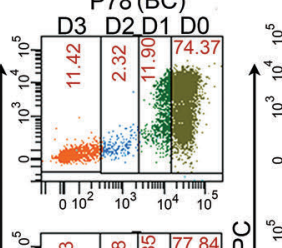

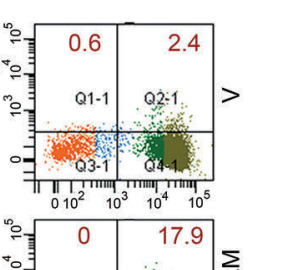

K $\cdot 100, \quad n=3$

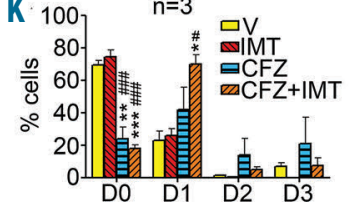

L

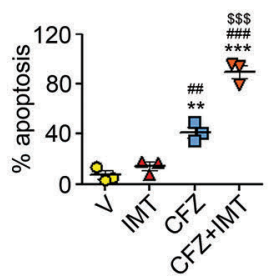

M CD34+ cells CD34+ cells

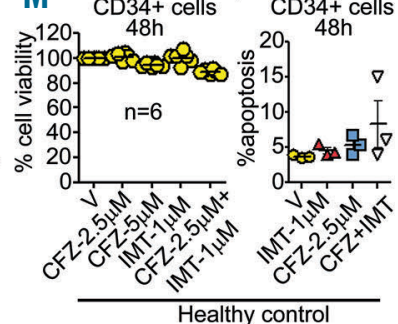

Figure 7. Clofazimine modulates PPAR y target gene expression and shows synergy with imatinib. (A) Clofazimine (CFZ) and pioglitazone (Pio) reduce signal transducer and activator of transcription 5 (STAT5) protein level in K562 cells (96 h). (B) Imatinib (IMT) but not CFZ or Pio (30 min) reduces STAT5-Y694 phosphorylation. (C) CFZ (5 $\mu \mathrm{M}, 24 \mathrm{~h}$ ) reduces STAT5B and BCL-2 transcripts in K562 cells. (D) IMT, but not CFZ or Pio (30 min), reduces CrkL-Y207 and BCR-ABL1 (ABL1-Y245) phosphorylation. (E) CFZ $(5 \mu \mathrm{M}, 24 \mathrm{~h})$ reduces STAT5B, hypoxia-inducible factor (HIF)- $1 \alpha$, HIF-2 $\alpha$ and CITED2 transcripts in CD34 ${ }^{+}$cells isolated from IMT-resistant chronic phase chronic myeloid leukemia (CP-CML) cells. (F) CFZ (72 h) shows superior cytotoxicity to thiazolidinediones in K562 cells. (G, H) CFZ (48 h) shows superior cytotoxic synergy with IMT in K562 cells (G) than Pio (H). (I) CFZ alone or in combination with IMT reduces colony-forming cells in soft agar assay (images in Online Supplementary Figure S2C $\mathrm{I}$; same set of data as Figure $1 \mathrm{H}$, with the addition of the CFZ+IMT group). (J-L) CFZ alone or in combination with IMT erodes the quiescent CD34+ population and induces apoptosis in these cells. The CD34+ population from IMT-resistant CP-CML cells $(n=3)$ were labeled with $2 \mu M$ carboxyfluorescein succidimidyl ester (CFSE) and treated as indicated $\left(96 \mathrm{~h}\right.$ ). Cells were gated on the basis of CFSE intensity. The distribution (\%) of CFSE/CD34 ${ }^{+}$cells in each cell division is shown by different colored dots (D0D3 represent the cell division number). Apoptosis in these cells was determined by annexin V staining. (J) Representative dot plots corresponding to patient 78 (P78) who was in blast crisis. (K) Cell numbers (\%) on DO-D3 from three patients (dot plots corresponding to other patients are presented in Online Supplementary Figure S12). (L) Percentage mean apoptosis from three patients whose data are plotted (see also Online Supplementary Figure S12). (M, N) CFZ alone or in combination with IMT does not alter viability (M) of CD34+ cells from healthy controls or induce apoptosis in these cells (N; dot plots in Online Supplementary Figure S2D). (M, N) Same set of data as in Figure $1 \mathrm{~K}$, with the addition of the CFZ+IMT group. Immunoblots are one representative of three independent experiments. Graphs are mean \pm standard error of mean of three independent experiments. ${ }^{*}, \# P<0.05, * *, \# \# P<0.01, * * *, \# \# \#, \$ \$ \$<0.001 . * V$ vs. treatment, ${ }^{\# I M T}$ vs. CFZ, ${ }^{s} \mathrm{CFZ}$ vs. IMT+CFZ. (C, E); Mann-Whitney $\mathrm{U}$ test. (I) Kruskal-Wallis test followed by the Dunn test. (K, L, N) One-way analysis of variance followed by the Bonferroni post test. 
A

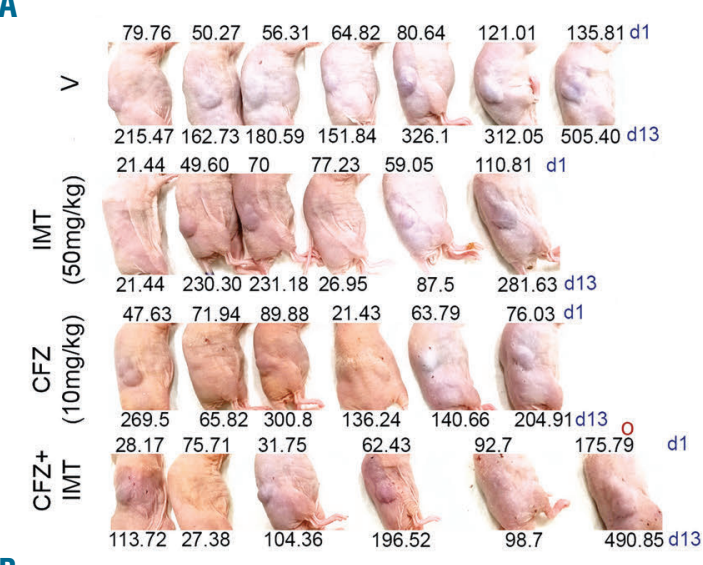

B

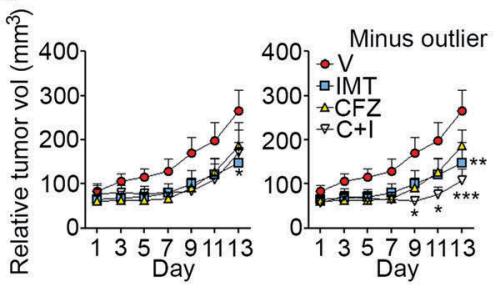

C
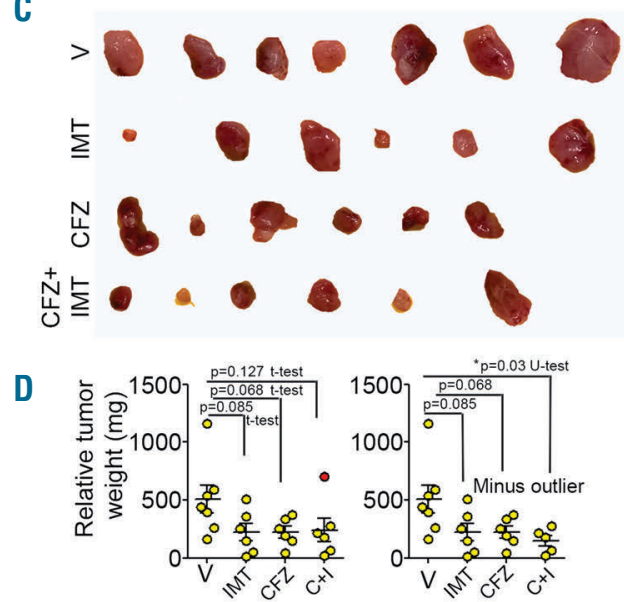

E

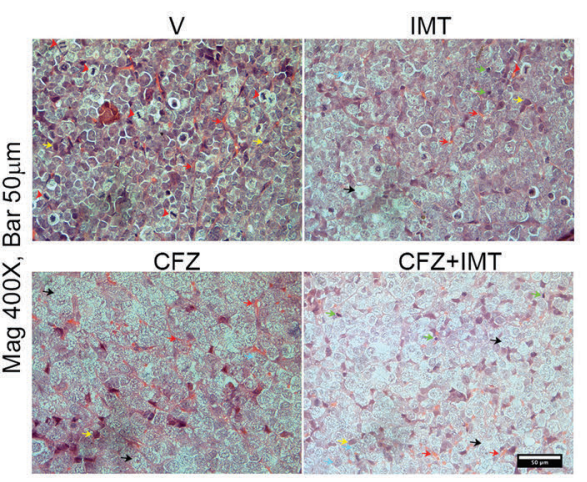

F

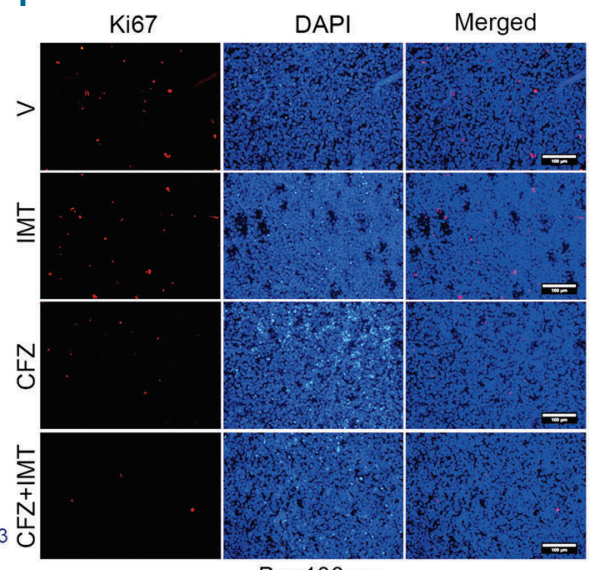

G

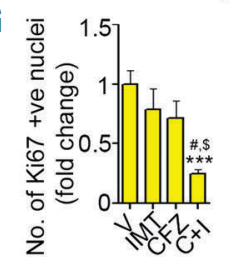

H TUNEL

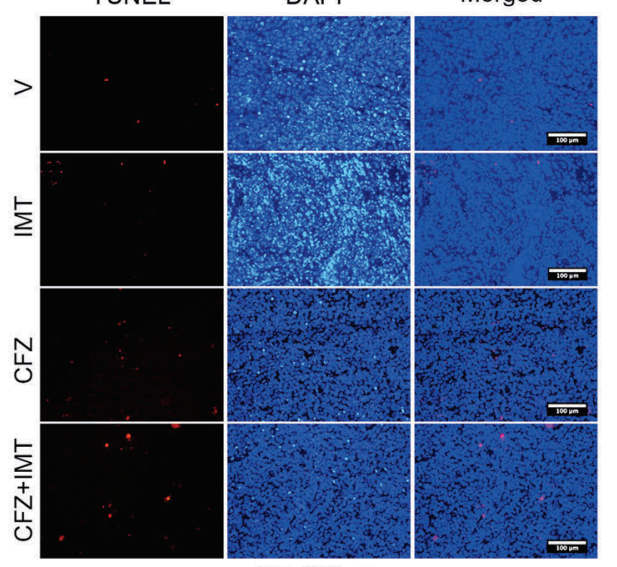

Bar $100 \mu \mathrm{m}$
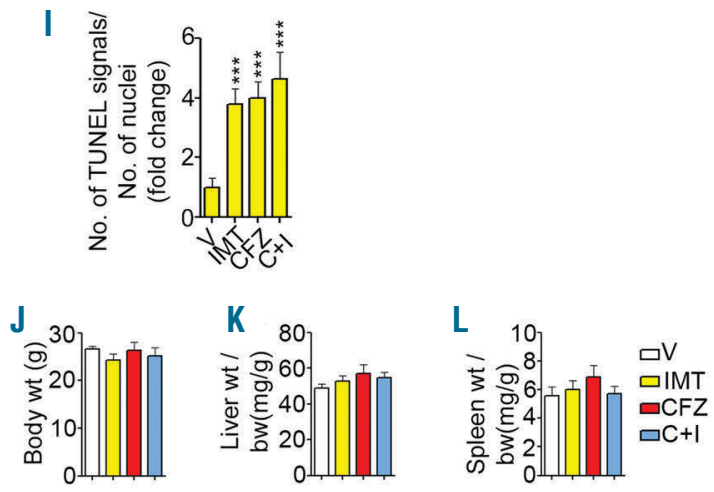

Figure 8. Effects of clofazimine and clofazamine + imatinib on K562 xenografts. (A) Photographs of mice with tumor, tumor volumes on day 1 (d1) and day 13 (d13) are given. The red ' $O$ ' represents an outlier based on d1 tumor volume $\left(>150 \mathrm{~mm}^{3}\right.$ ). (B) Relative tumor volume (day wise), left panel; all data included, right panel; minus the outlier (as shown in A). (C) Photographs of tumors. (D) Tumor weight, left panel; all data included (outlier marked in red); right panel: minus outlier. (E) Hematoxylin \& eosin staining of tumor sections (red arrowhead: mitotic cells; red arrow: vasculature; yellow arrow: myeloblasts; green arrow: pyknotic nuclei; cyan arrow; karyorrhexis; black arrow: degenerating cells). (F) Ki67 staining. (G) Number of Ki67-positive nuclei. (H) Terminal deoxynucleotidyl transferase dUTP nick end labeling (TUNEL) assay. (I) Number of TUNEL signals/ number of nuclei in a field. (E-I) Eighteen fields/group (6 animals/group). (J-L) Body weight (J), body weight normalized liver weight $(K)$, and body weight normalized spleen weight. (J-L) Vehicle; $n=7$, all treatment groups, $n=6$ per group. Microscopy was performed with a Leica DMI6000B microscope (Leica) and the findings were quantified with Image J software. (F, H) For counting, intensity of all images was enhanced in Microsoft office picture manager at a mid-tone setting of 80. Cropped images with uniformly increased brightness are given here for clarity; corresponding full-size images are presented in Online Supplementary Figure S13A, B. *,\#,\$P<0.05, **P<0.01, ***P<0.001. (B) Two-way analysis of variance (ANOVA), (J-L) One-way ANOVA followed by the Bonferroni post-test, (D) As indicated. (G, I) Kruskal-Wallis test followed by the Dunn test. 
and combining clofazimine with imatinib caused a further reduction (Figure 7I, Online Supplementary Figure S2C) (although the difference in effect of clofazimine vs. clofazimine + imatinib was not statistically insignificant, the $P$ values for the differences in effect between vehicle vs. clofazimine and for vehicle vs. clofazimine + imatinib were $P<0.01-0.001$ and $P<0.001$, respectively).

We next evaluated whether clofazimine alone or in combination with imatinib could erode quiescent LSC. To that prupose, we labeled CD34+ cells from imatinib-resistant patients (one patient in blast crisis) with carboxyfluorescein succidimidyl ester (CFSE) and treated them with the indicated drugs for $96 \mathrm{~h}$. While imatinib failed to reduce CFSE bight (non-dividing) cells, clofazimine alone or in combination with imatinib drastically reduced their number and increased the CFSE ${ }^{\text {dim }}$ (dividing cell) population (Figure 7J, K, Online Supplementary Figure S12). Evaluation of apoptosis in these cells revealed that clofazimine alone caused apoptosis in both CFSE ${ }^{\text {bight }}$ and CFSE ${ }^{\text {dim }}$ cells while combining clofazimine with imatinib caused their near obliteration (Figure 7L, Online Supplementary Figure S12). Clofazimine + imatinib did not affect normal $\mathrm{CD} 34^{+}$hematopoietic progenitors from healthy donors as clofazimine alone or in combination with imatinib caused $<14 \%$ loss of viability (Figure $7 \mathrm{M}$ ) and did not induce apoptosis in them (Figure 7N, Online Supplementary Figure S2D).

\section{Effect of clofazimine and the combination of clofazimine and imatinib in K562 xenografts}

To assess the effect of clofazimine, imatinib or their combination in vivo, athymic nude (nu/nu) mice harboring K562 xenografts were orally administered vehicle $(0.5 \%$ methyl cellulose), imatinib $(50 \mathrm{mg} / \mathrm{kg} /$ day; roughly equivalent to a human dose of $200 \mathrm{mg}$ ), clofazimine (10 $\mathrm{mg} / \mathrm{kg} /$ day, human equivalent dose of $50 \mathrm{mg}$ ) or a combination of clofazimine and imatinib $(10 \mathrm{mg} / \mathrm{kg} / \mathrm{day}$ and 50 $\mathrm{mg} / \mathrm{kg} /$ day, respectively) for 12 days. Analysis of tumor volume revealed a decreasing trend in all treatment groups which although not statistically significant (except for the imatinib group in which the tumor volume was statistically significantly reduced on day 13), became so after removal of an outlier (starting tumor volume $>150 \mathrm{~mm}^{3}$, marked as red ' $\mathrm{O}$ ', Figure 8A) in the clofazimine + imatinib group in which the reduction in tumor volume was statistically significant from day 9 onwards, while that in the imatinib group was statistically significant on day 13 only (Figure 8A, B). Analysis of tumor weight (tumor images in Figure $8 \mathrm{C}$ ) showed a similar pattern, with a decreasing trend in all treatment groups. However, upon removal of the outlier (marked red in Figure 8D, left panel and corresponding to the tumor shown in Figure 8A) a statistically significant reduction was present only in the clofazimine + imatinib group (Figure 8D). Histological analysis of the tumors revealed well-defined vasculature and a substantial number of mitotic cells in the group treated with vehicle, which were reduced in treatment groups, especially in the clofazimine + imatinib group (Figure 8E). Furthermore, karyopyknosis, karyorrhexis and degenerating cells were visible in treatment groups, especially in the clofazimine + imatinib group (Figure 8E). Staining of tumor sections for the cellular proliferation marker Ki67 revealed a significant reduction in the clofazimine + imatinib group, compared to the groups treated with vehicle or the individual drugs (Figure 8F, G). Determination of apoptotic cells by TUNEL staining revealed a significant enhancement of signals in all treatment groups (Figure $8 \mathrm{H}$, I). None of the animals in any of the treatment groups showed changes in body, liver or spleen weight (Figure 8JL). Together, these results indicate that combining clofazimine and imatinib causes a more robust anti-tumor activity than any of the drugs alone.

\section{Discussion}

Here, we identified clofazimine as an anti-CML agent that was particularly effective in cells from imatinib-resistant patients and robustly downregulated LSC including quiescent LSC. Clofazimine exerted its effect through PPAR $\gamma$. Recent evidence suggests that combining thiazolidinediones with tyrosine kinase inhibitors is an effective way to counter drug resistance in CML by eroding quiescent LSC that do not require BCR-ABL1 for survival..$^{1,45}$ Thiazolidinediones inhibit quiescent cells by transcriptional downregulation of STAT5, which is highly expressed in LSC, while imatinib regulates STAT5 phosphorylation. ${ }^{1}$ Combining both drugs thus causes stronger downregulation of STAT5 targets HIF-1 $\alpha$, HIF- $2 \alpha$ and CITED2, which are critical for LSC quiescence and maintenance. ${ }^{1}$ Here, we show that in addition to STAT5, clofazimine also regulated a novel pathway by modulating PPAR $\gamma$ ubiquitin ligase activity, which resulted in ROSdependent apoptosis via downregulation of PRDX1.

$P R D X 1$, originally cloned from K562 cells ${ }^{46}$ was initially described as a tumor suppressor ${ }^{47,48}$ but was later identified as an oncogene in various types of cancer in which its increased expression is associated with poor clinical outcome. ${ }^{49} P R D X 1 \mathrm{mRNA}$ was reported to be elevated in imatinib-resistant patients in whom no reduction in $B C R$ $A B L 1$ was co-related with higher PRDX1 transcript. $^{50} \mathrm{We}$ also observed a higher level of PRDX 1 transcripts in cells from CP-CML patients than in those from healthy donor, although the difference was not statistically significant. A significantly higher expression of PRDX1 was also observed in both CD34+38 and CD34+38+ LSC than in non-LSC, with the highest expression in CD $34^{+} 38^{+}$cells. Interestingly, PRDX1 is a secreted protein that enhances secretion of inflammatory cytokines by interacting with toll-like receptor $4 .{ }^{52,53}$ Clofazimine suppression of PRDX1 may thus explain its clinically observed anti-inflammatory functions ${ }^{6}$ and the anti-LSC activities seen here. Although PRDX1 has been well-studied in solid tumors, its function in CML is not clear and our study suggests that a detailed exploration of its role in CML progression will be important.

A plethora of reports implicate $M Y B$ in leukemogenesis which regulates factors such as c-Kit, ${ }^{54} \mathrm{CD} 34,{ }^{55}$ and FLT3, ${ }^{56}$ which are highly expressed in early progenitor cells and whose aberrant expression or mutations are associated with leukemia and poor clinical outcome..$^{5456}$ While MYB overexpression induces transformation in hematopoietic cells, ${ }^{57,58}$ its depletion inhibits colony growth in cells from CML patients including those in blast crisis. ${ }^{59,60}$ Here we found that clofazimine suppressed PRDX1 transcription by downregulating MYB expression and, for the first time, identified a specific MYB target sequence on the PRDX1 promoter.

$\mathrm{NF \kappa B}$ is one of the important downstream signaling pathways of the BCR-ABL1 oncoprotein. ${ }^{45,61}$ Abnormal 
NFkB activation has been reported in $\mathrm{CML}^{61}$ and LSC. ${ }^{62}$ Furthermore, p65 inhibition was shown to inhibit CML cells including those harboring the multidrug-resistant T315I BCR-ABL1 mutation. ${ }^{63,64}$ Our results show that clofazimine decreased p65 protein by increasing the ubiquitin ligase activity of PPAR $\gamma$. While thiazolidinediones increase PPAR $\gamma$ ubiquitin ligase activity at a suprapharmacological concentration of $\geq 100 \mu \mathrm{M}^{41}$ clofazimine was effective at a concentration of $5 \mu \mathrm{M}$. Clofazimine also displayed superior cytotoxic effects than thiazolidinediones. Furthermore, combining clofazimine with imatinib reduced the $\mathrm{IC}_{50}$ of imatinib by $>4$ logs whereas pioglitazone reduced it by 7 - to 10 -fold only. Combining imatinib with clofazimine in a K562 xenograft study caused greater reductions in tumor volume and weight than either drug alone, effects which were accompanied by substantially reduced proliferation and increased degenerative morphological changes in the group given combination therapy.

Pioglitazone is associated with cardiac and hepatic safety issues along with a significant risk of bladder cancer in users. ${ }^{3}$ That rosiglitazone does not increase the risk of bladder cancer ${ }^{3}$ indicates that this side effect is not associated with all PPAR $\gamma$ agonists. Being a phenazine derivative, clofazimine belongs to a different class of molecule. Given its superior efficacy over thiazolidinediones and that long-term assumption of clofazimine is not associated with major adverse effects; we propose clinical evaluation of clofazimine in combination with tyrosine kinase inhibitors in CML.

\section{Acknowledgments}

We dedicate this paper to the memory of Ranjan Kumar Bhagat. We thank Rune Toftgard, Odd Stokke Gabrielsen, Miguel Campanero, Chieko Kai, Giorgio Pochetti, David Mangelsdorf and Kimitoshi Kohno for kind gifts of plasmids. We thank Sharad Sharma and Madhav Nilkanth Mugale for help with histological analyses, and Dipak Datta and Jayanta Sarkar for useful discussions. SS acknowledges a mission-mode in-house project for cancer from CSIR, NC acknowledges funding from CSIR network project ASTHI (BSC 0201) and a grant-in-aid from the Department of Health Research-Indian Council of Medical Research (5/10/FR/5/2012-RHN/156), Government of India and AKT acknowledges funding from the CSIR network project INDEPTH. The authors acknowledge the sophisticated analytical instrument facility at CSIR-CDRI for FACS studies. $H K, S O S, S C$ and RK were supported by fellowships from the University Grants Commission. $A K S, S K, A G, S D, K L$ and $R M$ were supported by fellowships from CSIR. ND acknowledges the DBT-RA Program in Biotechnology and Life Sciences for a Fellowship. CDRI communication number: 9805.

\section{References}

1. Prost S, Relouzat F, Spentchian M, et al. Erosion of the chronic myeloid leukaemia stem cell pool by PPAR $\gamma$ agonists. Nature. 2015;525(7569):380-383.

2. Glodkowska-Mrowka E, Manda-Handzlik A, Stelmaszczyk-Emmel A, et al. PPAR $\gamma$ ligands increase antileukemic activity of second- and third-generation tyrosine kinase inhibitors in chronic myeloid leukemia cells. Blood Cancer J. 2016;6:e377.

3. Tuccori M, Filion $\mathrm{KB}$, Yin $\mathrm{H}$, Yu OH, Platt RW, Azoulay L. Pioglitazone use and risk of bladder cancer: population based cohort study. BMJ. 2016;352(i)1541.

4. Nissen SE, Wolski K. Effect of rosiglitazone on the risk of myocardial infarction and death from cardiovascular causes. N Engl J Med. 2007;356(24):2457-2471.

5. Gopal M, Padayatchi N, Metcalfe JZ, O'Donnell MR. Systematic review of clofazimine for the treatment of drug-resistant tuberculosis. Int $\mathrm{J}$ Tuberc Lung Dis. 2013;17(8):1001-1007.

6. Cholo MC, Steel HC, Fourie PB, Germishuizen WA, Anderson R. Clofazimine: current status and future prospects. J Antimicrob Chemother. 2012;67 (2):290-298.

7. Ren YR, Pan F, Parvez S, et al. Clofazimine inhibits human Kv1.3 potassium channel by perturbing calcium oscillation in T lymphocytes. PLoS One. 2008;3(12):e4009.

8. Leanza L, Henry B, Sassi N, et al. Inhibitors of mitochondrial Kv1.3 channels induce BAX/Bak-independent death of cancer cells. EMBO Mol Med. 2012;4(7):577-593.

9. Leanza L, Trentin L, Becker KA, et al. Clofazimine, Psora-4 and PAP-1, inhibitors of the potassium channel Kv1.3, as a new and selective therapeutic strategy in chronic lymphocytic leukemia. Leukemia. 2013;27 (8):1782-1785.

10. Smith GA, Tsui HW, Newell EW, et al Functional up-regulation of HERG K+ channels in neoplastic hematopoietic cells. J Biol Chem. 2002;277(21):18528-18534.

11. Schaad-Lanyi Z, Dieterle W, Dubois JP, Theobald W, Vischer W. Pharmacokinetics of clofazimine in healthy volunteers. Int J Lepr Other Mycobact Dis. 1987;55(1):9-15.

12. Yawalkar SJ, Vischer W. Lamprene (clofazimine) in leprosy. Basic information. Lepr Rev. 1979;50(2):135-144.

13. O'Connor R, O'Sullivan JF, O'Kennedy R. The pharmacology, metabolism, and chemistry of clofazimine. Drug Metab Rev. 1995;27(4):591-614

14. Marcato P, Dean CA, Giacomantonio CA Lee PW. Aldehyde dehydrogenase: its role as a cancer stem cell marker comes down to the specific isoform. Cell Cycle 2011;10(9):1378-1384.

15. Melemed AS, Ryder JW, Vik TA. Activation of the mitogen-activated protein kinase pathway is involved in and sufficient for megakaryocytic differentiation of CMK cells. Blood. 1997;90(9):3462-3470.

16. Fichelson S, Freyssinier JM, Picard F, et al. Megakaryocyte growth and development factor-induced proliferation and differentiation are regulated by the mitogen-activated protein kinase pathway in primitive cord blood hematopoietic progenitors. Blood. 1999;94(5):1601-1613.

17. Sardina JL, Lopez-Ruano G, Sanchez-Abarca LI, et al. p22phox-dependent NADPH oxidase activity is required for megakaryocytic differentiation. Cell Death Differ. 2010;17(12):1842-1854.

18. Nurhayati RW, Ojima Y, Nomura N, Taya M. Promoted megakaryocytic differentiation of K562 cells through oxidative stress caused by near ultraviolet irradiation. Cell Mol Biol Lett. 2014;19(4):590-600.

19. Chen S, Su Y, Wang J. ROS-mediated platelet generation: a microenvironmentdependent manner for megakaryocyte proliferation, differentiation, and maturation. Cell Death Dis. 2013;4:e722.

20. Shibayama-Imazu T, Sonoda I, Sakairi S, et al. Production of superoxide and dissipation of mitochondrial transmembrane potential by vitamin $\mathrm{K} 2$ trigger apoptosis in human ovarian cancer TYK-nu cells. Apoptosis. 2006;11(9):1535-1543

21. Dabrosin C, Ollinger K. Protection by alphatocopherol but not ascorbic acid from hydrogen peroxide induced cell death in normal human breast epithelial cells in culture. Free Radic Res. 1998;29(3):227-234.

22. Makpol S, Zainuddin A, Rahim NA, Yusof YA, Ngah WZ. Alpha-tocopherol modulates hydrogen peroxide-induced DNA damage and telomere shortening of human skin fibroblasts derived from differently aged individuals. Planta Med. 2010;76(9):869-875.

23. da Silveira Vargas F, Soares DG, Ribeiro AP, Hebling J, De Souza Costa CA. Protective effect of alpha-tocopherol isomer from vitamin $\mathrm{E}$ against the $\mathrm{H} 2 \mathrm{O} 2$ induced toxicity on dental pulp cells. Biomed Res Int. 2014;2014:895049.

24. Liou GY, Storz P. Reactive oxygen species in cancer. Free Radic Res. 2010;44(5):479-496.

25. Trachootham D, Alexandre J, Huang P. Targeting cancer cells by ROS-mediated mechanisms: a radical therapeutic approach? Nat Rev Drug Discov. 2009;8 (7):579-591.

26. Panieri E, Santoro MM. ROS homeostasis and metabolism: a dangerous liason in cancer cells. Cell Death Dis. 2016;7(6):e2253.

27. Shi X, Zhang Y, Zheng J, Pan J. Reactive oxygen species in cancer stem cells. Antioxid 
Redox Signal. 2012;16(11):1215-1228

28. Hofmann B, Hecht HJ, Flohe L. Peroxiredoxins. Biol Chem. 2002;383(34):347-364.

29. Ma Q. Role of nrf2 in oxidative stress and toxicity. Annu Rev Pharmacol Toxicol. 2013;53:401-426.

30. Kim JH, Bogner PN, Baek SH, et al. Up-regulation of peroxiredoxin 1 in lung cancer and its implication as a prognostic and therapeutic target. Clin Cancer Res. 2008;14(8):23262333.

31. Tanno B, Sesti F, Cesi V, et al. Expression of Slug is regulated by c-Myb and is required for invasion and bone marrow homing of cancer cells of different origin. J Biol Chem. 2010;285(38):29434-29445.

32. Wang OF, Lauring J, Schlissel MS. c-Myb binds to a sequence in the proximal region of the RAG-2 promoter and is essential for promoter activity in T-lineage cells. Mol Cell Biol. 2000;20(24):9203-9211.

33. Deng OL, Ishii S, Sarai A. Binding site analysis of c-Myb: screening of potential binding sites by using the mutation matrix derived from systematic binding affinity measurements. Nucleic Acids Res. 1996;24(4):766774.

34. Suhasini M, Pilz RB. Transcriptional elongation of c-myb is regulated by NF-kappaB (p50/RelB). Oncogene. 1999;18(51):73607369.

35. Pereira LA, Hugo HJ, Malaterre J, et al. MYB elongation is regulated by the nucleic acid binding of NFKB p50 to the intronic stemloop region. PLoS One. 2015;10(4): e0122919.

36. Toth CR, Hostutler RF, Baldwin AS, Jr., Bender TP. Members of the nuclear factor kappa B family transactivate the murine cmyb gene. J Biol Chem. 1995;270(13):76617671.

37. Lauder A, Castellanos A, Weston K. c-Myb transcription is activated by protein kinase $B$ (PKB) following interleukin 2 stimulation of Tcells and is required for PKB-mediated protection from apoptosis. Mol Cell Biol. 2001:21(17):5797-5805.

38. Campanero MR, Armstrong M, Flemington E. Distinct cellular factors regulate the $\mathrm{c}-\mathrm{myb}$ promoter through its E2F element. Mol Cell Biol. 1999;19(12):8442-8450.

39. Kim MY, Koh DI, Choi WI, et al. ZBTB2 increases PDK4 expression by transcriptional repression of RelA/p65. Nucleic Acids Res. 2015;43(3):1609-1625

40. Xu H, You M, Shi H, Hou Y. Ubiquitinmediated NFkB degradation pathway. Cell Mol Immunol. 2015;12(6):653-655

41. Hou Y, Moreau F, Chadee K. PPARy is an E3 ligase that induces the degradation of NFkB/p65. Nat Commun. 2012;3:1300.

42. Camacho IE, Serneels L, Spittaels K, Merchiers P, Dominguez D, De Strooper B. Peroxisome-proliferator-activated receptor gamma induces a clearance mechanism for the amyloid-beta peptide. J Neurosci. 2004:24(48):10908-10917.

43. Aprile M, Cataldi S, Ambrosio MR, et al PPAR $\gamma \delta 5$, a naturally occurring dominantnegative splice isoform, impairs PPAR $\gamma$ function and adipocyte differentiation. Cell Rep. 2018;25(6):1577-1592 e1576.

44. Liu JJ, Huang RW, Lin DJ, et al. Expression of survivin and bax/bcl-2 in peroxisome proliferator activated receptor-gamma ligands induces apoptosis on human myeloid leukemia cells in vitro. Ann Oncol. 2005;16(3):455-459.

45. Bitencourt R, Zalcberg I, Louro ID. Imatinib resistance: a review of alternative inhibitors in chronic myeloid leukemia. Rev Bras Hematol Hemoter. 2011;33(6):470-475

46. Sauri $\mathrm{H}$, Ashiian $\mathrm{PH}$, Kim AT, Shau $\mathrm{H}$ Recombinant natural killer enhancing factor augments natural killer cytotoxicity. Leukoc Biol. 1996;59(6):925-931.

47. Neumann CA, Krause DS, Carman CV, et al. Essential role for the peroxiredoxin $\operatorname{Prdx} 1$ in erythrocyte antioxidant defence and tumour suppression. Nature. 2003;424(6948):561565.

48. Cao J, Schulte J, Knight A, et al. Prdx1 inhibits tumorigenesis via regulating PTEN/AKT activity. EMBO J. 2009;28(10): 1505-1517.

49. Ding C, Fan X, Wu G. Peroxiredoxin 1 - an antioxidant enzyme in cancer. J Cell Mol Med. 2017;21(1):193-202.

50. Mascarenhas C, Woldmar L, Almeida MH, Andrade RV, Cunha AF, De Souza CA. Evaluation of peroxiredoxins (PRDX1, PRDX2 and PRDX6) expression in patients with chronic myeloid leukemia (CML) treated with imatinib in first line. Blood. 2014;124(21):5545-5545.

51. Nieborowska-Skorska M, Kopinski PK, Ray $\mathrm{R}$, et al. Rac2-MRC-cIII-generated ROS cause genomic instability in chronic myeloid leukemia stem cells and primitive progenitors. Blood. 2012:119(18):4253-4263.

52. Riddell JR, Wang XY, Minderman H, Gollnick SO. Peroxiredoxin 1 stimulates secretion of proinflammatory cytokines by binding to TLR4. J Immunol. 2010;184 (2):1022-1030.

53. Liu $\mathrm{CH}$, Kuo SW, Hsu LM, et al. Peroxiredoxin 1 induces inflammatory cytokine response and predicts outcome of cardiogenic shock patients necessitating extracorporeal membrane oxygenation: an observational cohort study and translational approach. J Transl Med. 2016;14(1):114.

54. Ratajczak MZ, Perrotti D, Melotti P, et al $M y b$ and ets proteins are candidate regulators of c-kit expression in human hematopoietic cells. Blood. 1998;91(6):19341946.

55. Melotti P, Ku DH, Calabretta B. Regulation of the expression of the hematopoietic stem cell antigen CD34: role of c-myb. J Exp Med. 1994:179(3):1023-1028.

56. Volpe G, Walton DS, Del Pozzo W, et al $\mathrm{C} / \mathrm{EBP} \alpha$ and MYB regulate FLT3 expression in AML. Leukemia. 2013;27(7):1487-1496.

57. Lutwyche JK, Keough RA, Hughes TP Gonda TJ. Mutation screening of the c-MYB negative regulatory domain in acute and chronic myeloid leukaemia. Br J Haematol. 2001;114(3):632-634.

58. Bussolari R, Candini O, Colomer D, et al. Coding sequence and intron-exon junctions of the c-myb gene are intact in the chronic phase and blast crisis stages of chronic myeloid leukemia patients. Leuk Res. 2007;31(2):163-167.

59. Ratajczak MZ, Hijiya N, Catani L, et al. Acute- and chronic-phase chronic myelogenous leukemia colony-forming units are highly sensitive to the growth inhibitory effects of c-myb antisense oligodeoxynucleotides. Blood. 1992;79(8):1956-1961.

60. Calabretta B, Sims RB, Valtieri $M$, et al Normal and leukemic hematopoietic cells manifest differential sensitivity to inhibitory effects of c-myb antisense oligodeoxynucleotides: an in vitro study relevant to bone marrow purging. Proc Natl Acad Sci U S A. 1991;88(6):2351-2355

61. Kirchner D, Duyster J, Ottmann O, Schmid RM, Bergmann L, Munzert G. Mechanisms of BCR-ABL1-mediated NF-kappaB/Rel activation. Exp Hematol. 2003;31(6):504-511.

62. Guzman ML, Neering SJ, Upchurch D, et al. Nuclear factor-kappaB is constitutively activated in primitive human acute myelogenous leukemia cells. Blood. 2001;98(8): 2301-2307.

63. Lounnas N, Frelin C, Gonthier N, et al. NFkappaB inhibition triggers death of imatinib-sensitive and imatinib-resistant chronic myeloid leukemia cells including T315I BCR-ABL1 mutants. Int J Cancer. 2009;125(2):308-317.

64. Lu Z, Jin Y, Chen C, Li J, Cao O, Pan J. Pristimerin induces apoptosis in imatinibresistant chronic myelogenous leukemia cells harboring T315I mutation by blocking NF-kappaB signaling and depleting BCRABL1. Mol Cancer. 2010;9:112. 